\title{
EnKF Assimilation of High-Resolution, Mobile Doppler Radar Data of the 4 May 2007 Greensburg, Kansas, Supercell into a Numerical Cloud Model
}

\author{
Robin L. TANAmAChi, ${ }^{*,+, \#}$ Louis J. Wicker, ${ }^{@}$ DAVID C. Dowell, ${ }^{\&}$ Howard B. Bluestein, ${ }^{\#}$ \\ DANIEL T. DAWSON II, ${ }^{+,}$AND MING XUE* \\ * Center for Analysis and Prediction of Storms, University of Oklahoma, Norman, Oklahoma \\ + Cooperative Institute for Mesoscale Meteorological Studies, Norman, Oklahoma \\ \# School of Meteorology, University of Oklahoma, Norman, Oklahoma \\ @ National Severe Storms Laboratory, Norman, Oklahoma \\ \& NOAA/Earth Systems Research Laboratory, Boulder, Colorado
}

(Manuscript received 3 April 2012, in final form 10 July 2012)

\begin{abstract}
Mobile Doppler radar data, along with observations from a nearby Weather Surveillance Radar-1988 Doppler (WSR-88D), are assimilated with an ensemble Kalman filter (EnKF) technique into a nonhydrostatic, compressible numerical weather prediction model to analyze the evolution of the 4 May 2007 Greensburg, Kansas, tornadic supercell. The storm is simulated via assimilation of reflectivity and velocity data in an initially horizontally homogeneous environment whose parameters are believed to be a close approximation to those of the Greensburg supercell inflow sector. Experiments are conducted to test analysis sensitivity to mobile radar data availability and to the mean environmental near-surface wind profile, which was changing rapidly during the simulation period. In all experiments, a supercell with similar location and evolution to the observed storm is analyzed, but the simulated storm's characteristics differ markedly. The assimilation of mobile Doppler radar data has a much greater impact on the resulting analyses, particularly at low altitudes $(\leq 2 \mathrm{~km})$, than modifications to the near-surface environmental wind profile. Differences in the analyzed updrafts, vortices, cold pool structure, rear-flank gust front structure, and observation-space diagnostics are documented. An analyzed vortex corresponding to the enhanced Fujita scale 5 (EF-5) Greensburg tornado is stronger and deeper in experiments in which mobile (higher resolution) Doppler radar data are included in the assimilation. This difference is linked to stronger analyzed horizontal convergence, which in turn is associated with increased stretching of vertical vorticity. Changing the near-surface wind profile appears to impact primarily the updraft strength, availability of streamwise vorticity for tilting into the vertical, and lowlevel vortex strength and longevity.
\end{abstract}

\section{Introduction}

Radar is one of few atmospheric measurement tools capable of collecting volumetric data resolving substormscale features. Assimilation of radar data into numerical weather prediction (NWP) models to improve understanding of convective storm dynamics is now a fairly routine exercise, and analysis and prediction of highimpact, substorm-scale features such as tornadoes is a natural objective. Numerous studies have been undertaken in this area in the past two decades; summaries of these

Corresponding author address: Robin L. Tanamachi, Center for Analysis and Prediction of Storms, University of Oklahoma, 120 David L. Boren Blvd., Suite 2500, Norman, OK 73072.

E-mail: rtanamachi@ou.edu efforts are provided by Lilly (1990), Sun (2005), Kain et al. (2010), and Stensrud et al. (2009).

Weather Surveillance Radar-1988 Doppler (WSR88D) data are now routinely collected across most of the contiguous United States. The two measured radar variables most often assimilated into NWP models are Doppler velocity $V_{r}$ and radar reflectivity factor $Z$. NWP models require and calculate additional state variables (e.g., temperature and pressure) that observations of $V_{r}$ and $Z$ do not furnish. While a Doppler radar can provide detailed velocity information within convective storms, the cross-beam components of velocity are unobserved and must be calculated or inferred. The quantity $Z$ is integrated over many different hydrometeors with variable scattering properties (Doviak and Zrnić 1993), and has nonlinear 
relationships with model state variables related to hydrometeors (Snyder and Zhang 2003; Dowell et al. 2011). The ensemble Kalman filter (EnKF; Evensen 1994; Houtekamer and Mitchell 1998) provides an attractive pathway toward inferring unobserved model state variables based, at least in part, on Doppler radar measurements (Snyder and Zhang 2003; Dowell et al. 2004a,b; French et al. 2006; Jung et al. 2008a; Aksoy et al. 2009; Dowell and Wicker 2009; Dowell et al. 2011; Snook et al. 2011).

Owing to the scanning geometry and wide geographic spacing of the WSR-88D (more than $200^{2} \mathrm{~km}^{2}$ ), substantial regions of the atmosphere remain unobserved at altitudes below $1 \mathrm{~km}$ AGL, where weather events directly impact human activity (National Research Council 1995). Zhang et al. (2004) found that assimilation of radial velocity observations at and below $2 \mathrm{~km}$ AGL improved analyzed cold pool structure. Snook et al. (2011) demonstrated that assimilation of $Z$ and $V_{r}$ data from short-range, stationary, X-band Collaborative Adaptive Sensing of the Atmosphere (CASA) radars, in addition to data from a WSR-88D, greatly impacted analyzed lowlevel wind fields in a 2007 tornadic mesoscale convective system. In particular, the analyzed locations of nearsurface wind features such as gust fronts and misovortices were improved via assimilation of shallow volumes of CASA data. Previous studies have indicated that the locations of such near-surface features may be crucial to tornadogenesis (Marquis et al. 2008; Wurman et al. 2010; Markowski et al. 2011b; Marquis et al. 2012).

In this study, we assimilate $V_{r}$ data from an X-band, mobile Doppler radar in addition to WSR-88D $Z$ and $V_{r}$ data into the National Severe Storms Laboratory (NSSL) Collaborative Model for Multiscale Atmospheric Simulation (NCOMMAS; Wicker and Skamarock 2002; Coniglio et al. 2006), and assess the impact on storm-scale EnKF analyses of the 4 May 2007 Greensburg, Kansas, tornadic supercell (hereafter "the Greensburg storm"). This historic storm produced at least 22 tornadoes over a period of 9 h, including an enhanced Fujita scale 5 (EF-5) tornado that devastated the town of Greensburg at 02450250 UTC (Lemon and Umscheid 2008; Bluestein 2009), causing 11 fatalities. The University of Massachusetts mobile, X-band, polarimetric Doppler radar (UMass XPol; Bluestein et al. 2007) captured much of the Greensburg storm's early evolution, collecting data in at least 10 tornadoes (Tanamachi et al. 2012). Since the UMass X-Pol was located closer to the hook echo of the Greensburg storm and collected data at lower levels than the nearest WSR-88D, this dataset provides an unusual opportunity to examine the impacts of assimilating mobile Doppler radar data on EnKF analyses of a supercell.
Mobile Doppler radar can potentially move close to a target storm and sample an area of interest near the surface. While data from mobile X-band Doppler radars have been assimilated via EnKF into NWP models in previous studies (French 2006; Marquis et al. 2010, 2012), only one other study is known to the authors in which data from a mobile Doppler radar and WSR-88D data were assimilated together (Marquis et al. 2010). In that study, as in this one, radar data were assimilated into an initially horizontally homogeneous environment with a flat model bottom boundary. The Greensburg storm occurred over rather flat terrain (southwest Kansas), so we consider our use of a flat model bottom boundary reasonable.

The locations of stationary radars and their orientation in space are generally well documented. Such radars are sited on tall platforms to minimize beam blockage, and clutter patterns around the radars become known to the data users with time. In contrast, assimilation of data from mobile Doppler radars poses additional challenges. The mobile radar may be deployed under challenging conditions (e.g., in great haste, on uneven surfaces, or in severe weather), and its orientation may not be well documented. This issue has been mitigated to some extent through the use of global positioning system (GPS) devices, which can record the radar location to within $10 \mathrm{~m}$, and hydraulic leveling systems, which can ensure a level antenna base to within $\pm 0.2^{\circ}$. In addition, because the radar antenna is height constrained for transportability, the transmitted radar signal is more susceptible to blockage by trees, hills, buildings, and telephone poles. Ground clutter patterns around the radar change with every deployment. The clutter around the antenna may be documented via photographs, site surveys, maps, and/or the radar data. Usually, as in this case, extensive quality control must be applied to mobile Doppler radar data before they can be assimilated into an NWP model.

Our study also addresses the relative impact on analyses of modification of an initial boundary layer wind profile. Bluestein (2009) speculated that an intensifying low-level jet in the Greensburg storm's environment may have played a role in the formation and intensification of the Greensburg tornado. Do changes to the wind profile impact the analyzed storms and accompanying vortices? For how long does the model "remember" an initial wind profile, once radar data are assimilated? These questions were partially addressed in a companion study (Dawson et al. 2012) that focused on the impact of modifications to the boundary layer wind profile on forecasts of the Greensburg storm and its vortices. In this study, we focus on how changes to the boundary layer wind profile impact the analyses. 
KDDC Reflectivity, 1.0 km AGL

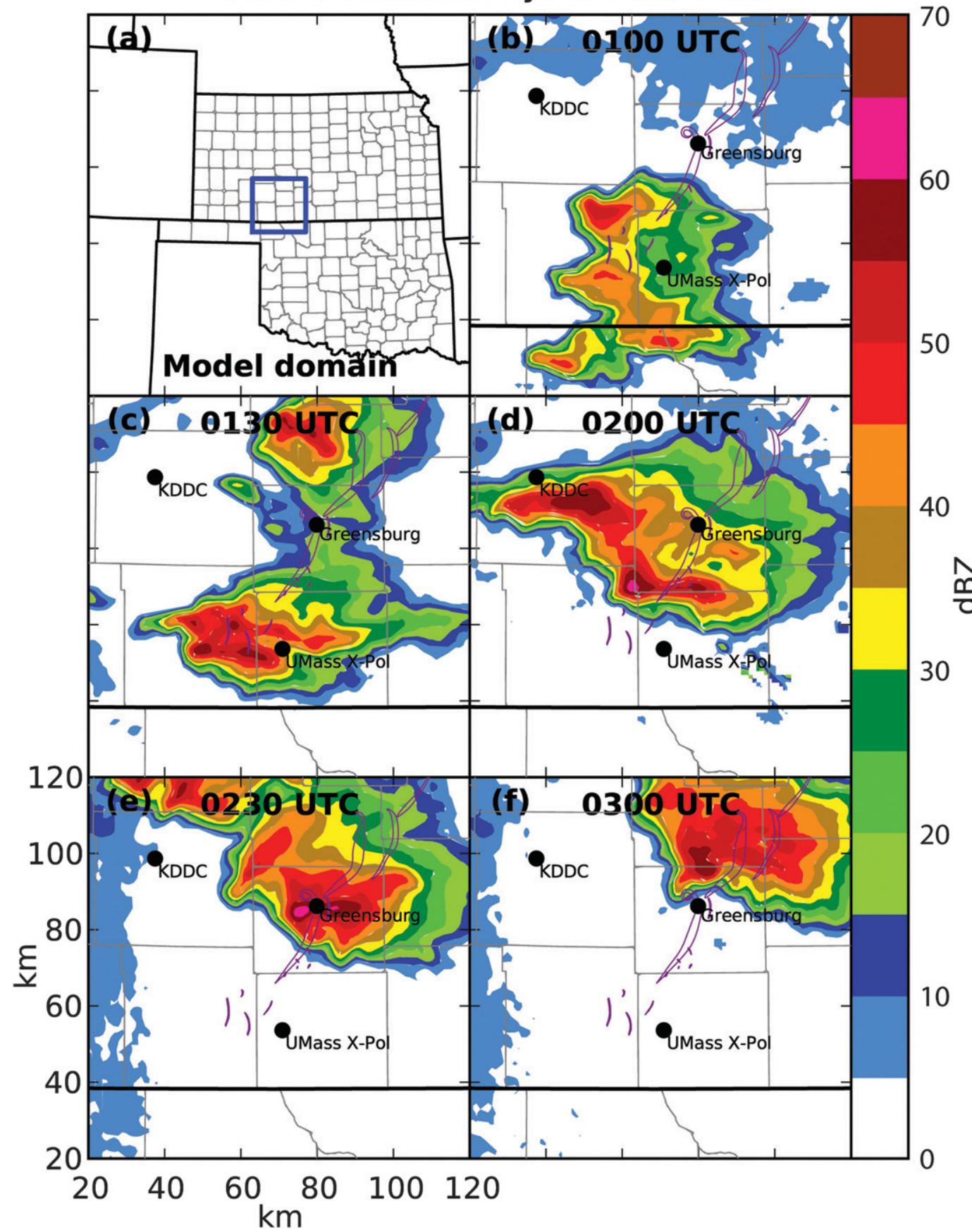

FIG. 1. (a) Model domain (outlined in blue) and objectively analyzed KDDC reflectivity (dBZ) at $1.0 \mathrm{~km}$ AGL within that domain at (b) 0100, (c) 0130, (d) 0200, (e) 0230, and (f) 0300 UTC 5 May 2007. Distances (km) are relative to the southwest corner of the model domain. Thin gray (heavy black) lines denote county (state) boundaries. Surveyed tornado damage tracks (outlined in purple) are courtesy of J. Hutton of the NWS forecast office in Dodge City, KS.

We briefly summarize the meteorological background of the Greensburg storm and the radar data used in this study in the next section (section 2). Section 3 details our NCOMMAS experiments, including the development of the initial model environment, experiment parameters, radar data quality control, and objective analysis. Experiment results, which show substantial impacts from both mobile radar data 


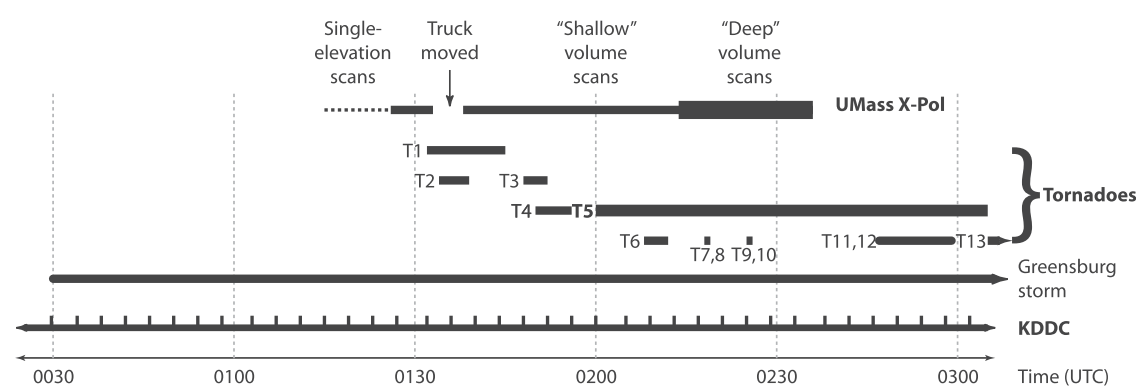

FIG. 2. Timeline of Greensburg storm, tornadoes, and radar data collection. Start times of KDDC volumes are shown as tick marks. Tornadoes are numbered chronologically [following Lemon and Umscheid (2008)]. For UMass X-Pol deployment times, dashed lines indicate times when single-elevation scans were collected; the solid line indicates when "shallow" volume scans were collected; and the thick bar indicates when "deep" volume scans were collected.

assimilation and modification of the initial wind profile, are presented in section 4 . Section 5 summarizes the study.

\section{The 4 May 2007 Greensburg, Kansas, storm and radar data}

Because the Greensburg storm has been well documented by Lemon and Umscheid (2008), Monfredo (2008), Bluestein (2009), and Tanamachi et al. (2012), only a brief review of its early evolution will be given here. The Greensburg storm formed at 0030 UTC on an outflow boundary from a previous storm, became a mature supercell over the next hour (Fig. 1), and produced four EF-0 and EF-1 tornadoes (tornadoes 14) from 0132 to 0155 UTC (Fig. 2). Tornado 5 (the Greensburg tornado) formed at 0200 UTC, generated a 53-km-long damage path, inflicted EF-5 damage in Greensburg from 0245 to 0250 UTC, and finally dissipated at 0305 UTC. The Greensburg tornado was accompanied by at least seven smaller, satellite tornadoes (6-12) from 0210 to 0259 UTC (Lemon and Umscheid 2008). Although this storm produced several additional significant tornadoes, we are primarily concerned with the time span (through 0300 UTC) covering the first 10 in the sequence (Tanamachi et al. 2012).

In this study, only radar data were assimilated, not only to limit the complications introduced by the addition of other data sources, but also because few supplemental data were available. (Those observations were used to specify the initial model environment, as described below.) We assimilated $Z$ and $V_{r}$ data from the WSR-88D in Dodge City, Kansas (KDDC), and, in some experiments, $V_{r}$ data from the UMass X-Pol (Bluestein et al. 2007; Tanamachi et al. 2012). Some relevant characteristics of these two radars are shown in Table 1.

\section{a. WSR-88D data}

The Greensburg tornado occurred at a range of $\sim 60 \mathrm{~km}$ from the WSR-88D at the National Weather Service (NWS) forecast office at KDDC (Table 1; Fig. 3). KDDC collected data continuously at 4.1-min intervals in the Greensburg storm, including its initiation at $0030 \mathrm{UTC}^{1}$ In volume coverage pattern 12 (VCP12; Brown et al. 2005), a WSR-88D executes a "step spiral" scanning pattern, cycling through 14 elevation angles ranging from $0.5^{\circ}$ to $19.5^{\circ}$. We used 37 volumes of KDDC $Z$ and $V_{r}$ data, from 0029 to 0302 UTC. Because the data exhibited velocity aliasing, they were manually dealiased using National Center for Atmospheric Research (NCAR) Solo II radar data editing and visualization software (Oye et al. 1995).

\section{b. UMass X-Pol data}

As part of an ongoing severe weather research effort, UMass X-Pol (Bluestein et al. 2007) was deployed $48 \mathrm{~km}$ south-southwest of Greensburg, and collected data in the Greensburg storm continuously from 0112 to 0233 UTC (apart from one 6-min break when the truck had to be moved in order to minimize beam blockage to the west; Fig. 2). The UMass X-Pol azimuthal sector (around $90^{\circ}$ wide in most instances) was rotated clockwise toward the north to follow the target storm. Initially, the crew collected shallow volumes $\left(3^{\circ}-10^{\circ}\right)$, but switched to deeper volumes $\left(3^{\circ}-15^{\circ}\right.$ or $\left.3^{\circ}-20^{\circ}\right)$ as tornado 5 matured. A complete description of the deployment can be found in Tanamachi et al. (2012).

\footnotetext{
${ }^{1}$ The Greensburg storm was also detected by KVNX and the WSR-88D located at the NWS forecast office in Amarillo, Texas (KAMA), among others. The distance between the Greensburg tornado and KVNX was $\sim 130 \mathrm{~km}$; for KAMA this distance was $\sim 330 \mathrm{~km}$.
} 
TABLE 1. Characteristics of KDDC and UMass X-Pol radars in 2007.

\begin{tabular}{lcc}
\hline \hline \multicolumn{1}{c}{ Radar } & KDDC & UMass \\
& (WSR-88D) & X-Pol \\
\hline Type & Stationary & Mobile \\
Wavelength $(\mathrm{cm})$ & 10 & 3 \\
Half-power beamwidth & $1.0^{\circ}$ & $1.2^{\circ}$ \\
Peak transmitted power $(\mathrm{kW})$ & 475 & 25 \\
Max unambiguous range $(\mathrm{km})$ & 231 & 75 \\
Max unambiguous velocity $\left(\mathrm{m} \mathrm{s}^{-1}\right)$ & 32.5 & 19.2 \\
Range gate spacing & $1 \mathrm{~km}(Z)$, & $150 \mathrm{~m}$ \\
& $250 \mathrm{~m}\left(v_{r}\right)$ & \\
Max azimuthal scan rate $\left(\mathrm{s}^{-1}\right)$ & $30^{\circ}$ & $24^{\circ}$ \\
Polarimetry & Single & Dual \\
\hline
\end{tabular}

\section{Experiment setup}

\section{a. Background}

The experiment setup is derived from that used by Aksoy et al. (2009), who simulated different types of isolated convective storms via assimilation of WSR-88D $Z$ and $V_{r}$ observations on storm-scale domains (i.e., with horizontal dimensions $\sim 160-200 \mathrm{~km}$ ) with flat bottom boundaries. They initialized their model runs with horizontally homogeneous environments derived from the nearest (in both space and time) available rawinsonde observation (sounding), populating their initial ensembles by adding perturbations to the wind profiles in these soundings to account for uncertainty in the rawinsonde measurements.

Some discussion about the choice of a horizontally homogeneous model initial environment (also known as a "single-sounding environment") is warranted. Stensrud and Gao (2010), who also performed radar data assimilation (DA) experiments on the Greensburg storm, demonstrate the value of realistic $3 \mathrm{D}$ variability in a model initial environment for 1-h forecasts. They conclude that "knowledge of horizontal environmental variability is important to successful convective-scale ensemble predictions

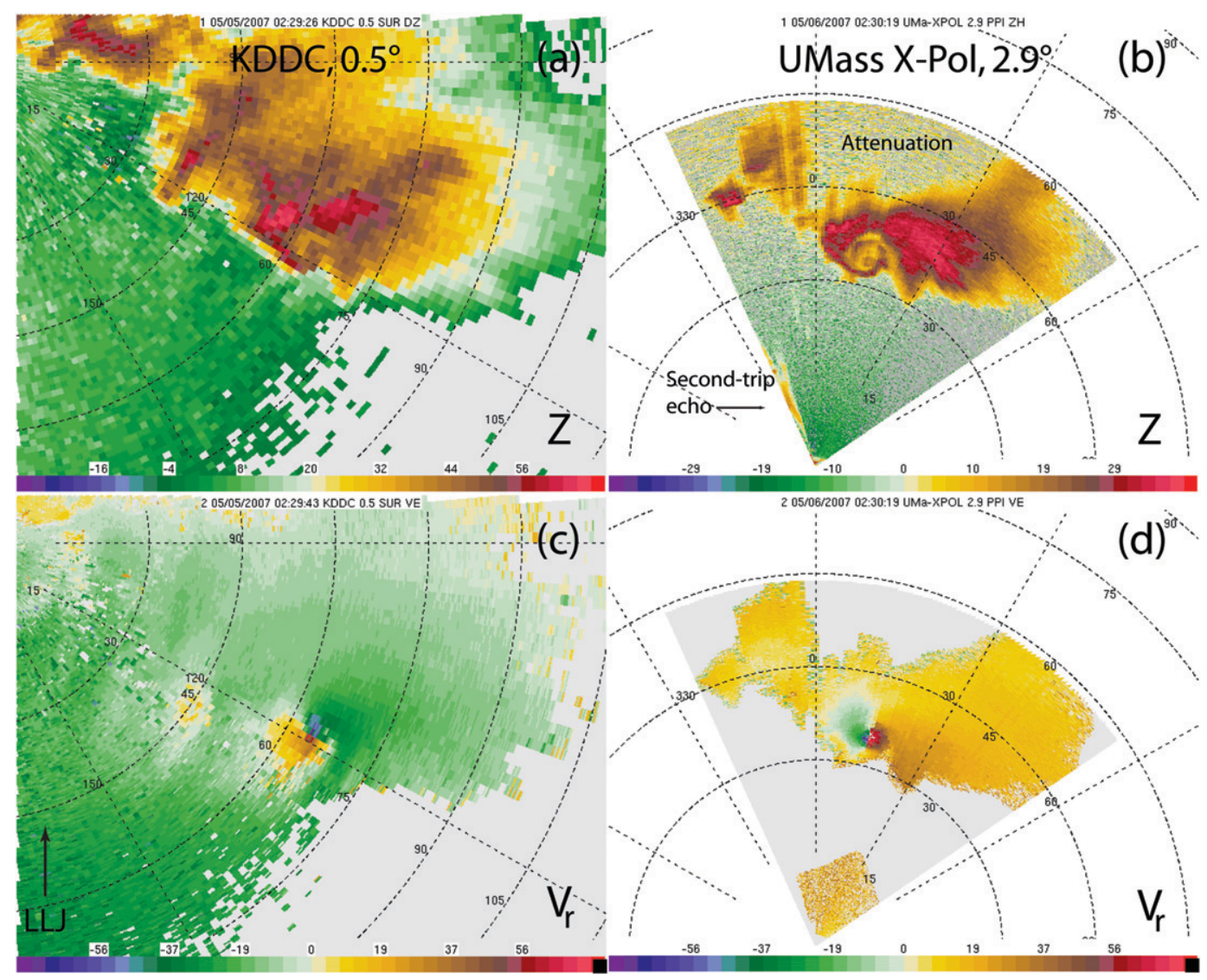

FIG. 3. Side-by-side comparisons of (a),(b) $Z$ and (c),(d) $V_{r}$ data collected at 0230 UTC (when tornado 5 was mature) by (a),(c) KDDC at $0.5^{\circ}$ and (b),(d) UMass X-Pol at $2.9^{\circ}$. All panels use the same scale and are centered on the vortex. Range rings are every $15 \mathrm{~km}$. UMass X-Pol reflectivity was attenuated on the north side of the Greensburg storm because of large hail in the storm core; $V_{r}$ data in this region were manually excluded. The $V_{r}$ data were manually dealiased. UMass X-Pol $V_{r}$ data were further edited to exclude "noisy" velocity data outside the storm, but some gates close to the radar, which contain information about the near-surface wind fields, were retained. 


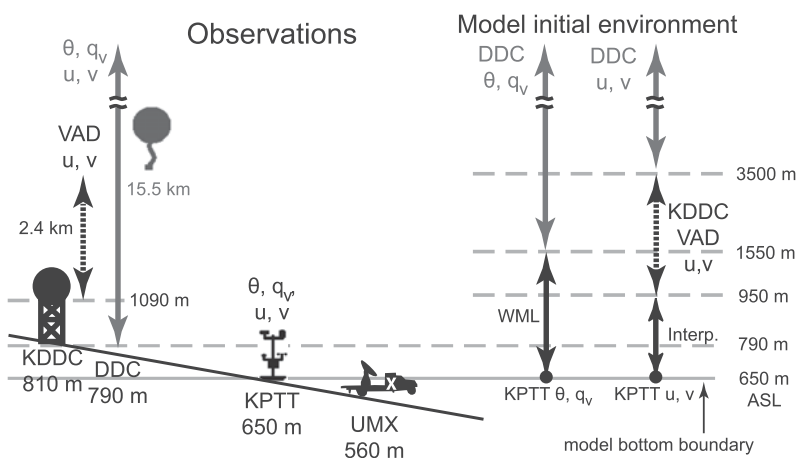

FIG. 4. Observations used in the construction of the model initial environment. Heights and distances between observing platforms are not to scale.

and needs to be included in real-data experiments." In light of these results, Dawson et al. (2012) ruminate on the merits of a horizontally homogeneous model initial environment, and argue that the single-sounding approach makes sensitivity studies more straightforward to interpret. Here we focus on assessing the analyses' sensitivity to assimilation of different radar datasets and different initial wind profiles, whereas Stensrud and Gao (2010) focus on prediction of the Greensburg storm.

Considering that the Greensburg storm developed on an outflow boundary from a previous storm (Bluestein 2009), it is accepted that there will be some errors in the analyses resulting from initial environmental horizontal inhomogeneity that is not accounted for. The model environment is only horizontally homogeneous at the initial time; forward integration of the model and DA make the model states horizontally inhomogeneous at all subsequent times.

\section{b. Model initial environment}

The initial environment used in this study (Fig. 4), thought to approximate the inflow environment of the Greensburg storm, was developed in tandem with that used by Dawson et al. (2012); therefore, many similarities can be seen between their initial environment and ours.

The nearest available rawinsonde observation (in both space and time) to the Greensburg storm was that collected by NWS at 0000 UTC 5 May 2007 at Dodge City, Kansas (DDC). This rawinsonde was launched after a dryline passage substantially modified both the wind and thermodynamic profiles below about $800 \mathrm{hPa}$; they were certainly not representative of the inflow region of the Greensburg storm. In addition, between 0000 and $0300 \mathrm{UTC}$, an intensifying low-level jet (LLJ) was observed in velocity-azimuth display (VAD) wind profiles from KDDC (Fig. 5); this temporal variability was not captured by the single DDC rawinsonde. We chose

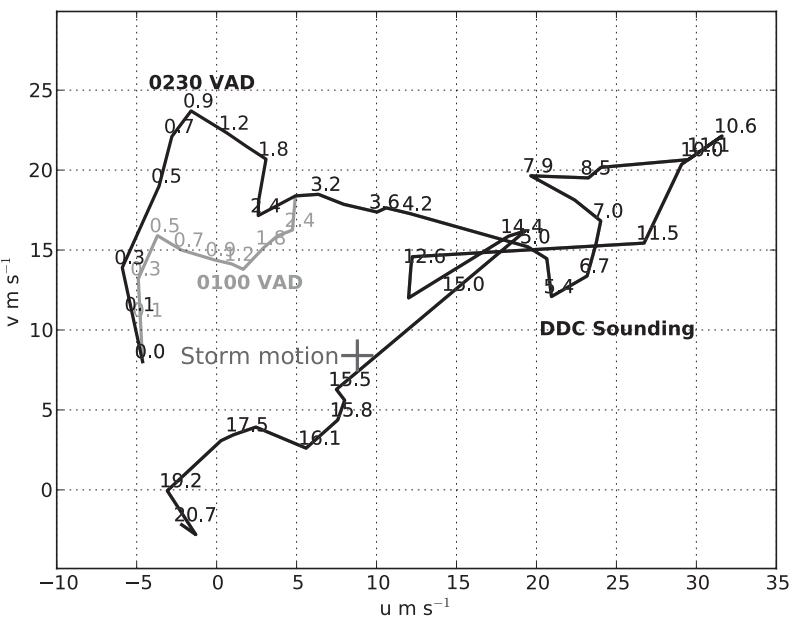

FIG. 5. Hodographs of the initial wind profiles used in the EnKF experiments prior to interpolation to the model grid levels. The black (gray) curve depicts the "vad0230" ("vad0100") wind profile. Greensburg storm motion is denoted by a dark gray cross. Altitude labels are in $\mathrm{km}$ AGL. The surface velocity components are from the KPTT observation at 0210 UTC 5 May 2007, while those in the $0.3-3.0-\mathrm{km}$ layer are from KDDC VAD retrievals. The wind profiles above $3.0 \mathrm{~km}$ are from the 0000 UTC 5 May 2007 DDC sounding and are identical for both experiments.

to retain the DDC thermodynamic and wind profiles aloft, but made modifications to the near-surface layers to account for some of the known temporal and spatial variability of the near-storm environment.

From 0000 to 0300 UTC, the nearest well-calibrated, automated surface observation station (ASOS) to the Greensburg storm is sited at Pratt, Kansas (KPTT), $49 \mathrm{~km}$ east of Greensburg. ${ }^{2}$ The forward-flank region of the Greensburg storm began to pass over KPTT at 0230 UTC. We assumed that the closest prior KPTT observation (taken at $0210 \mathrm{UTC} ; T=26^{\circ} \mathrm{C}, T_{d}=$ $19^{\circ} \mathrm{C}, u=-5 \mathrm{~m} \mathrm{~s}^{-1}, v=8 \mathrm{~m} \mathrm{~s}^{-1}$ ), was representative of the near-surface inflow environment of the Greensburg storm. With no more detailed information available about the thermodynamic characteristics of the boundary layer, we simply inserted a 900-m-deep, well-mixed (constant $\theta=307 \mathrm{~K}$, constant $q_{v}=15 \mathrm{~g} \mathrm{~kg}^{-1}$ ) layer. The presence of such a well-mixed boundary layer is supported by the rawinsonde observation taken at Lamont, Oklahoma, at 0000 UTC 5 May 2007 (not shown), in which a nearly well-mixed layer extended from the surface (317 m MSL) to $1500 \mathrm{~m}$ MSL.

\footnotetext{
${ }^{2}$ Data from Kansas Groundwater Management District 5 evapotranspiration stations, including one located on the north side of Greensburg, were reported as hourly averages (S. Falk 2009, personal communication) and were not suitable for use in these experiments.
} 

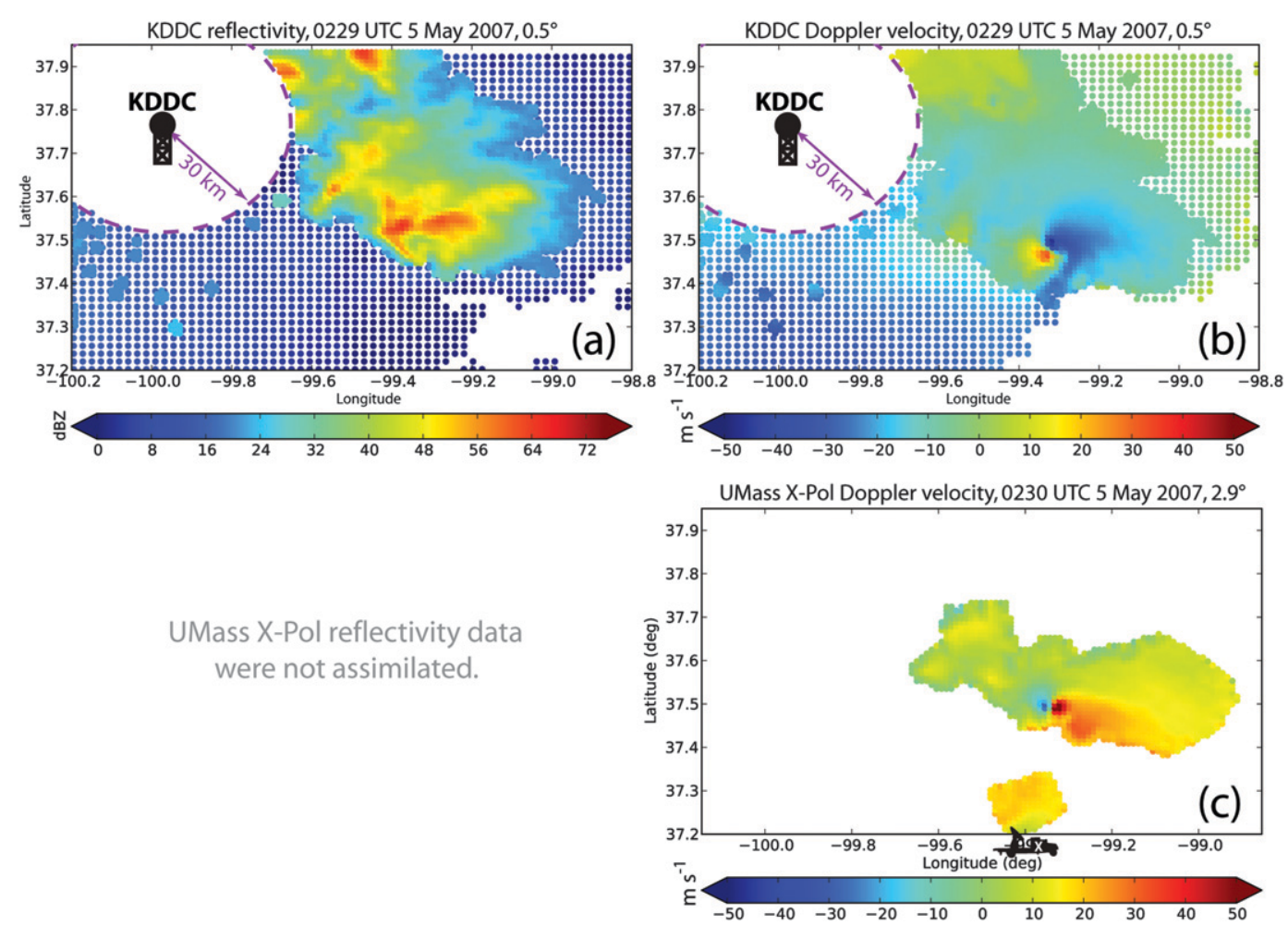

FIG. 6. Objectively analyzed KDDC (a) reflectivity $(\mathrm{dBZ})$ and (b) Doppler velocity $\left(\mathrm{m} \mathrm{s}^{-1}\right)$ collected at $0229 \mathrm{UTC}$ at an elevation angle of $0.5^{\circ}$, and (c) objectively analyzed UMass X-Pol Doppler velocity $\left(\mathrm{m} \mathrm{s}^{-1}\right.$ ) collected at $0230 \mathrm{UTC}$ at an elevation angle of $2.9^{\circ}$. KDDC observations associated with reflectivity values greater than or equal to (less than) $20 \mathrm{dBZ}$ were analyzed at $1-\mathrm{km}(2 \mathrm{~km})$ grid spacing. The dashed purple circle denotes the 30-km range ring around KDDC; data inside this radius were discarded for the lowest three elevation angles $\left(0.5^{\circ}, 0.9^{\circ}\right.$, and $\left.1.3^{\circ}\right)$ in each volume in order to avoid ground clutter targets.

To test the sensitivity of the analyses to the low-level wind profiles in much the same manner as Dawson et al. (2012), the VAD (Browning and Wexler 1968) technique was used to retrieve wind profiles from KDDC $V_{r}$ measurements taken at 0100 and 0230 UTC, and these wind profiles were then inserted between the surface and $3000 \mathrm{~m} \mathrm{AGL}{ }^{3}$ The Greensburg storm was organizing at 0100 UTC, when the LLJ was weaker, while at 0230 UTC the Greensburg EF-5 tornado was mature, and the LLJ was strengthening. The lowest useable VAD wind retrieval (at $1090 \mathrm{~m}$ MSL, $300 \mathrm{~m}$ above KDDC) was linearly interpolated to the KPTT surface velocity observation.

The resulting initial model environment is supportive of supercell thunderstorms, with $4600 \mathrm{~J} \mathrm{~kg}^{-1}$ of CAPE (Dawson et al. 2012, see their Fig. 1) and $26 \mathrm{~m} \mathrm{~s}^{-1}$ (50 kt) of 0-6-km bulk shear (Fig. 5).

\footnotetext{
${ }^{3}$ Attempts to retrieve boundary layer wind profiles from UMass $\mathrm{X}$-Pol data using VAD were unsuccessful because of the narrow $\left(\leq 90^{\circ}\right)$ azimuthal sector used.
}

\section{c. Software}

We used NCOMMAS to generate the ensemble of short-term (3 min) forecasts required by EnKF (see Table 2). NCOMMAS is a compressible, nonhydrostatic numerical weather prediction model designed to simulate atmospheric events in a simplified framework (Coniglio et al. 2006; Dowell and Wicker 2009). The model employs a third-order Runge-Kutta advection scheme with a secondary, shorter time step for acoustic modes (Wicker and Skamarock 2002). We assimilated radar data using the ensemble square root filter (EnSRF; Whitaker and Hamill 2002; Dowell et al. 2004b) implementation in NCOMMAS (Dowell and Wicker 2009).

Radar observations were assimilated into NCOMMAS as a stream of point observations. The localization response function (Gaspari and Cohn 1999) for each observation goes to zero at a horizontal (vertical) radius of $6.0(3.0) \mathrm{km}$. The observation operator for $Z$ is described in the appendix of Dowell et al. (2011). The observation operator for $V_{r}$ is 
TABLE 2. List of model parameters.

\begin{tabular}{|c|c|}
\hline Parameter & Value \\
\hline Model initial time & 0030 UTC 5 May 2007 \\
\hline Assimilation window & 0100-0300 UTC 5 May 2007 \\
\hline $\begin{array}{l}\text { Assimilation cycle } \\
\text { frequency }\end{array}$ & $3 \mathrm{~min}$ \\
\hline Ensemble members & 45 \\
\hline Simulation domain & $140 \mathrm{~km} \times 140 \mathrm{~km} \times 20 \mathrm{~km}$ \\
\hline Domain size & $141 \times 141 \times 41$ \\
\hline Center of domain & $37.58^{\circ} \mathrm{N}, 99.22^{\circ} \mathrm{W}$ \\
\hline Model bottom boundary & $650 \mathrm{~m} \mathrm{MSL}$ \\
\hline Horizontal grid spacing & $1 \mathrm{~km}$ \\
\hline Vertical grid spacing & $\begin{array}{l}500 \mathrm{~m} \text { (first scalar level } \\
250 \mathrm{~m} \mathrm{AGL})\end{array}$ \\
\hline Cloud microphysical scheme & $\begin{array}{l}\text { Lin-Farley-Orville } \\
\quad \text { (Gilmore et al. 2004) }\end{array}$ \\
\hline Rain density $\rho_{r}$ & $1000 \mathrm{~kg} \mathrm{~m}^{-3}$ \\
\hline Rain intercept parameter $N_{0 r}$ & $8.0 \times 10^{5} \mathrm{~m}^{-4}$ \\
\hline Hail/graupel density $\rho_{h}$ & $800 \mathrm{~kg} \mathrm{~m}^{-3}$ \\
\hline $\begin{array}{l}\text { Hail/graupel intercept } \\
\text { parameter } N_{0 \mathrm{~h}}\end{array}$ & $4.0 \times 10^{4} \mathrm{~m}^{-4}$ \\
\hline Snow density $\rho_{s}$ & $100 \mathrm{~kg} \mathrm{~m}^{-3}$ \\
\hline Snow intercept parameter $N_{0 \mathrm{~s}}$ & $3.0 \times 10^{6} \mathrm{~m}^{-4}$ \\
\hline Lateral boundaries & Open \\
\hline Model time step & $3 \mathrm{~s}$ \\
\hline $\begin{array}{l}\text { Assumed observation } \\
\text { error variance } \\
\text { for } Z\left(\sigma_{z}^{2}\right) \text { and } V_{r}\left(\sigma_{\mathrm{vr}}^{2}\right)\end{array}$ & $(5.0 \mathrm{~dB} Z)^{2},\left(3.0 \mathrm{~m} \mathrm{~s}^{-1}\right)^{2}$ \\
\hline
\end{tabular}

$$
v_{r}=\left(\sin \alpha \cos \theta_{e}\right) u+\left(\cos \alpha \cos \theta_{e}\right) v+\left(\sin \theta_{e}\right)\left(w-w_{t}\right),
$$

where $\alpha$ and $\theta_{e}$ are the azimuth and elevation angles of the radar beam, respectively; $(u, v, w)$ is the model air velocity interpolated to the observation location; and $w_{t}$ is the fall speed of precipitation particles within the grid volume.

\section{d. Radar data editing and objective analysis}

To reduce the number of radar observations $\left(\sim 10^{5}-\right.$ $10^{6}$ for a single radar sweep) to a manageable quantity for assimilation, both the $\operatorname{KDDC}\left(Z\right.$ and $\left.V_{r}\right)$ and UMass X-Pol ( $V_{r}$ only) data from 0030 to 0302 UTC were objectively analyzed to the model horizontal grid spacing using the Cressman (1959) technique. The radar data were analyzed so that each sweep remained on its original, conical sweep surface while being horizontally interpolated to the grid (Sun and Crook 2001; Dowell et al. 2004b), thereby retaining the greater data density in the vertical near the radars.

The KDDC data covered the entire horizontal extent of the model domain. Areas in which KDDC $Z$ was greater than or equal to (less than) $20 \mathrm{~dB} Z$ were analyzed at $1 \mathrm{~km}(2 \mathrm{~km})$ horizontal grid spacing with a radius of influence of $1.5 \mathrm{~km}(3 \mathrm{~km}$; e.g., Figs. $6 \mathrm{a}, \mathrm{b})$.

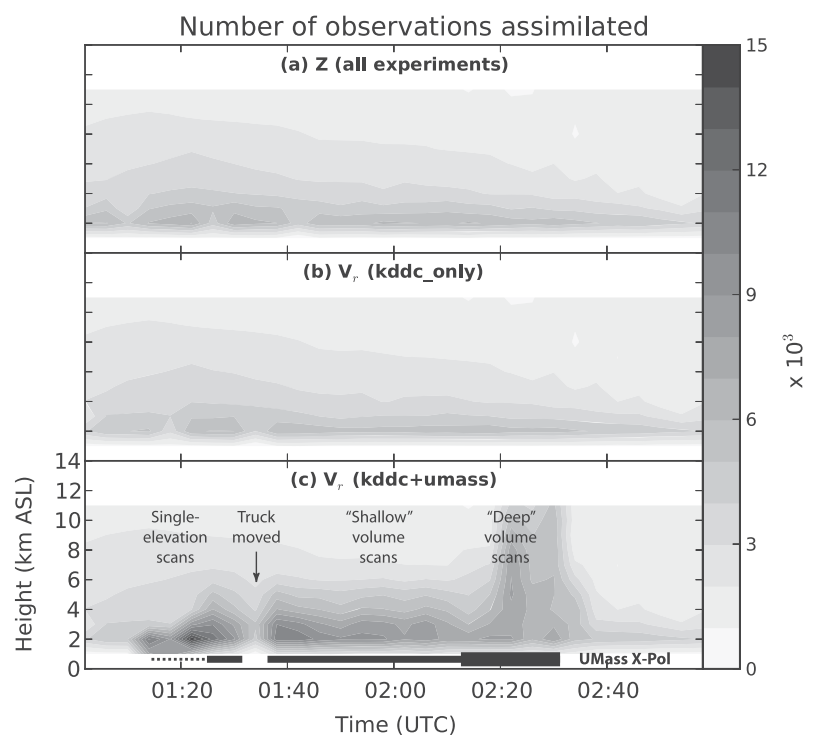

FIG. 7. Number of (a) $Z$ and (b),(c) $V_{r}$ observations (e.g., Fig. 6) available for assimilation as a function of time and altitude. UMass $\mathrm{X}$-Pol $Z$ observations are not shown because they were not assimilated; the $Z$ observations are only from KDDC and are the same for all experiments. Times when UMass X-Pol collected shallow and deep volumes (Fig. 2) are delineated in (c). Observations are plotted in 4-min bins for clarity. The movement out of the domain of the Greensburg storm (as well as other storms) explains the overall decrease with time of both $\mathrm{KDDC} Z$ and $V_{r}$ observations.

These "low reflectivity" ( $<20 \mathrm{~dB} Z)$ observations were analyzed at a coarser horizontal grid spacing so as to reduce the total number of observations being assimilated, while still retaining enough information in areas of low $Z$ (where, presumably, little to no convection is ongoing) to suppress spurious convection in the model (Caya et al. 2005; Tong and Xue 2005; Aksoy et al. 2009; Dowell et al. 2011).

Assimilation of KDDC data from nonmeteorological targets was problematic in preliminary versions of these experiments. In some cases, the relatively high $Z$ values associated with clutter targets (such as wind farms) were erroneously recast by the data assimilation system as convective precipitation. To mitigate this issue, KDDC $Z$ and $V_{r}$ data in the lowest three elevation angles $\left(0.5^{\circ}\right.$, $0.9^{\circ}$, and $1.3^{\circ}$ ) within $30 \mathrm{~km}$ of KDDC were omitted from the objective analysis (e.g., Figs. 6a,b). This practice had the undesirable effect of removing some observations of real convective precipitation within $30 \mathrm{~km}$ of KDDC, at altitudes at or below $680 \mathrm{~m}$ AGL. However, this convective precipitation (which occurred well away from the Greensburg storm) was not the focus of these experiments, and observations at higher elevation angles helped to mitigate the effects of these omitted low-altitude data.

UMass X-Pol $Z$ data exhibited an attenuation "shadow" from the Greensburg storm's hail core resulting from 
Ens. Mean Reflectivity, 05 May 2007 02:00:00, 750 m AGL

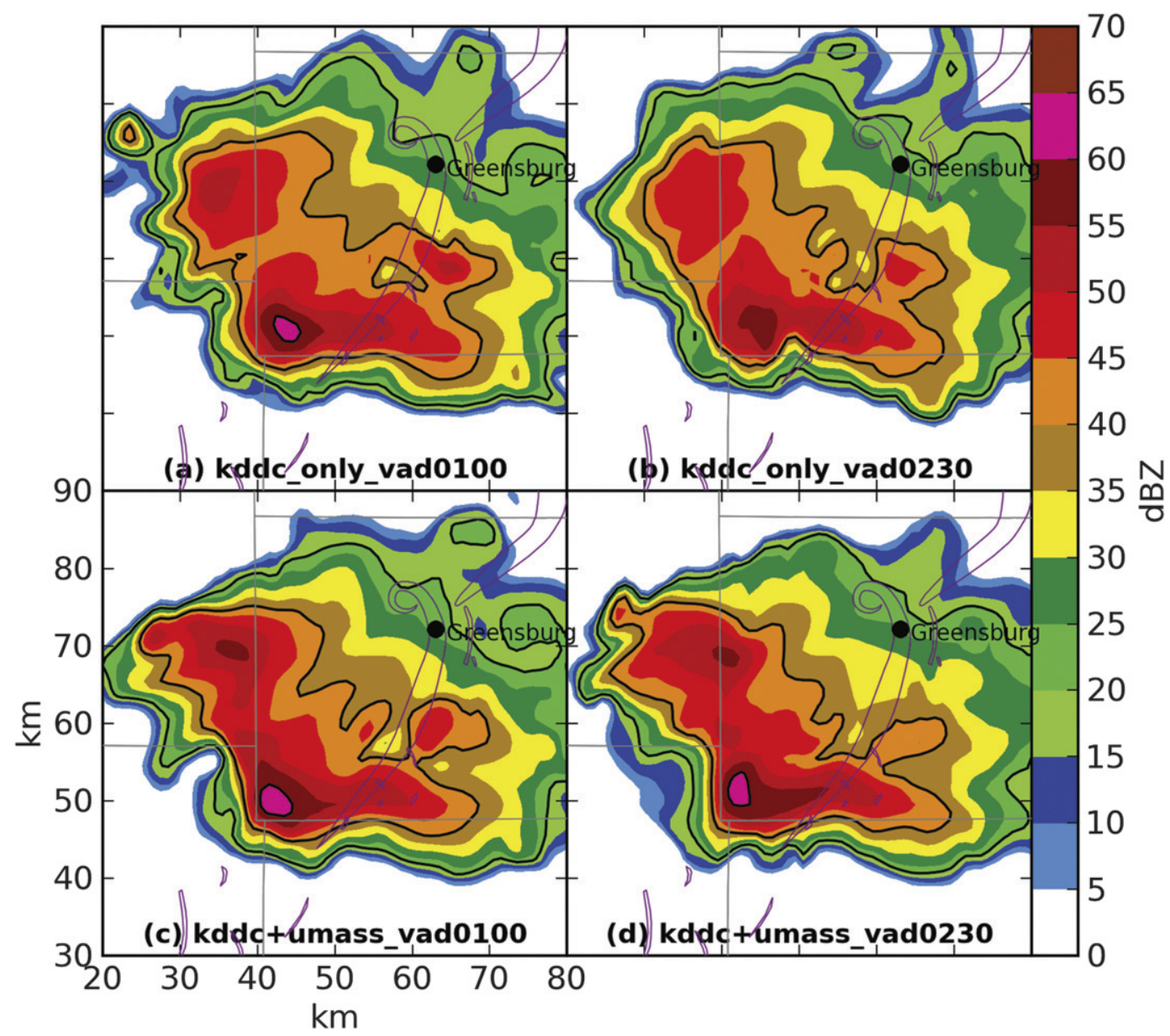

FIG. 8. Prior ensemble mean reflectivity $(\mathrm{dBZ})$ at $0.8 \mathrm{~km}$ AGL at $0200 \mathrm{UTC}$ (when the Greensburg tornado formed) for experiments (a) kddc_only_vad0100, (b) kddc_only_vad0230, (c) kddc+umass_vad0100, and (d) kddc+ umass_vad0230. County boundaries are drawn in gray; tornado damage tracks are outlined in purple.

Mie scattering of the X-band signal by large $(>4-\mathrm{cm}$ diameter) hail. [In accordance with Doviak and Zrnić (1993), we use units of equivalent radar reflectivity, $\mathrm{dB} Z_{e}$, for the UMass $\mathrm{X}-\mathrm{Pol} Z$ data.] In addition, these data were not well calibrated for this deployment (Tanamachi et al. 2012). For these reasons, UMass X-Pol $Z$ data were not assimilated; assimilating them would likely have had the undesired effect of suppressing convection in the supercell. UMass X-Pol $V_{r}$ data associated with uncalibrated $Z$ values less than $-18 \mathrm{~dB} Z_{e}$ and that appeared to contain primarily noise (low $Z$ observations, areas of attenuation) were manually discarded. ${ }^{4}$

\footnotetext{
${ }^{4}$ We experimented with several reflectivity and SNR thresholds, but found that manual editing was the most reliable way to retain the desired data near UMass X-Pol while discarding undesired data associated with second trip echo, clutter, and attenuation.
}

These data would be objectively analyzed as near-zero velocity, when in fact there is simply no reliable information about the velocities in those areas. The resulting UMass X-Pol $V_{r}$ field contained only data from the Greensburg storm, and some boundary layer observations near UMass X-Pol (e.g., Fig. 6c).

\section{e. Model configuration}

The experiment setup is summarized in Table 2. The domain, which had 1-km horizontal grid spacing, was centered slightly southeast of Greensburg (Fig. 1) and was sufficiently large to contain most of the storm between 0100 and 0300 UTC. While a grid with $1-\mathrm{km}$ horizontal spacing is not generally sufficient to resolve a tornado, the Greensburg tornado was exceptionally wide, with a maximum damage track width of $2.1 \mathrm{~km}$ (Lemon and Umscheid 2008). As will be seen, the 


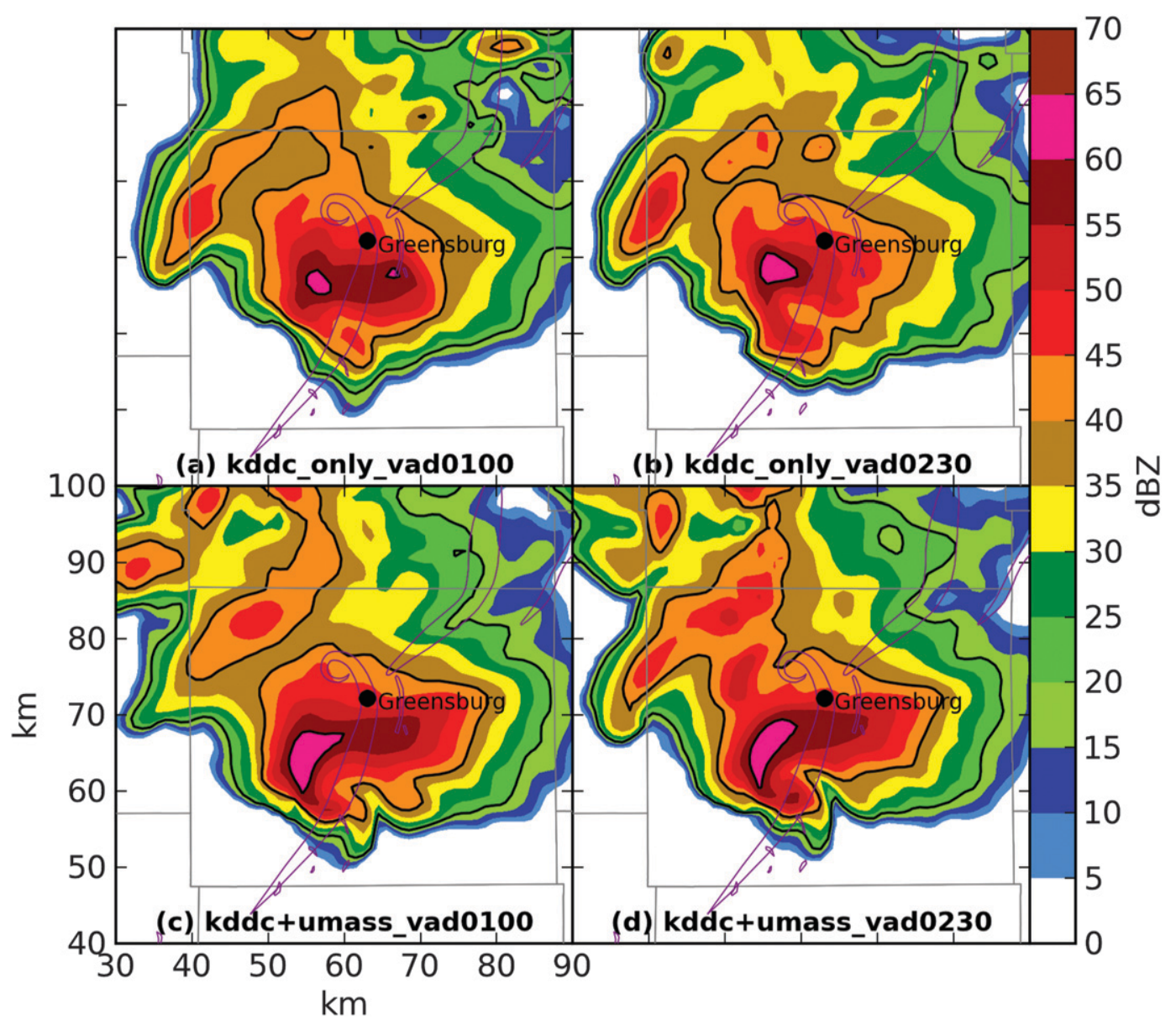

FIG. 9. As in Fig. 8, but at 0230 UTC (when the Greensburg tornado was mature).

minimally resolved Greensburg tornado, its associated mesocyclone, and a number of other vortices corresponding to smaller tornadoes are distinct in the resulting model fields.

Within the NCOMMAS simplified experimental framework, no surface fluxes, turbulence parameterizations, or radiation physics were used. For cloud and precipitation microphysics, we used the Gilmore et al. (2004) version of the Lin et al. (1983) parameterization scheme. This single-moment scheme uses five hydrometeor classes, including three ice classes (cloud ice, snow, and hail/graupel). Large hail was documented in the Greensburg storm (National Climatic Data Center 2009; Lemon and Umscheid 2008), so relatively high hail/graupel density $\left(\rho_{h}=800 \mathrm{~kg} \mathrm{~m}^{-3}\right)$ and low slope intercept parameter $\left(N_{0 \mathrm{~h}}=4 \times 10^{4} \mathrm{~m}^{-4}\right)$ were prescribed. It has been found in previous idealized simulations of supercell thunderstorms that a large intercept parameter for rain [e.g., $N_{0 \mathrm{r}}=8 \times 10^{6} \mathrm{~m}^{-4}$ from Marshall and Palmer (1948), which has been used in many studies] biases the rain distribution toward small drops and can result in unrealistically strong cold pools owing to enhanced evaporation (Snook and Xue 2008; Dawson et al. 2010). Since the presence of large raindrops in the hook and forward-flank regions was inferred from high UMass X-Pol $Z_{\mathrm{DR}}$ measurements (Tanamachi et al. 2012), the intercept parameter for rain $N_{0 \mathrm{r}}$ was prescribed as $8 \times 10^{5} \mathrm{~m}^{-4}$.

To populate the initial (0100 UTC) ensemble of 45 members, and also to account for instrument and representativeness error in the DDC sounding and KDDC VADs used to generate the base-state environment, random, normally distributed wind components $\sim N\left(\bar{v}, \sigma_{v}\right)=$ $N\left(0 \mathrm{~m} \mathrm{~s}^{-1}, 2.0 \mathrm{~m} \mathrm{~s}^{-1}\right)$ were added to each level of each ensemble member's base-state wind profile (Dowell et al. 2011). The temperature profile (identical in all experiments) was not perturbed so as to avoid inadvertent generation of superadiabatic layers. Small $[7.5 \mathrm{~km}(1.5 \mathrm{~km})$ horizontal (vertical) radius, $2.0 \mathrm{~K}$ ] thermal bubbles were then added to each member below $5 \mathrm{~km}$ AGL in regions 


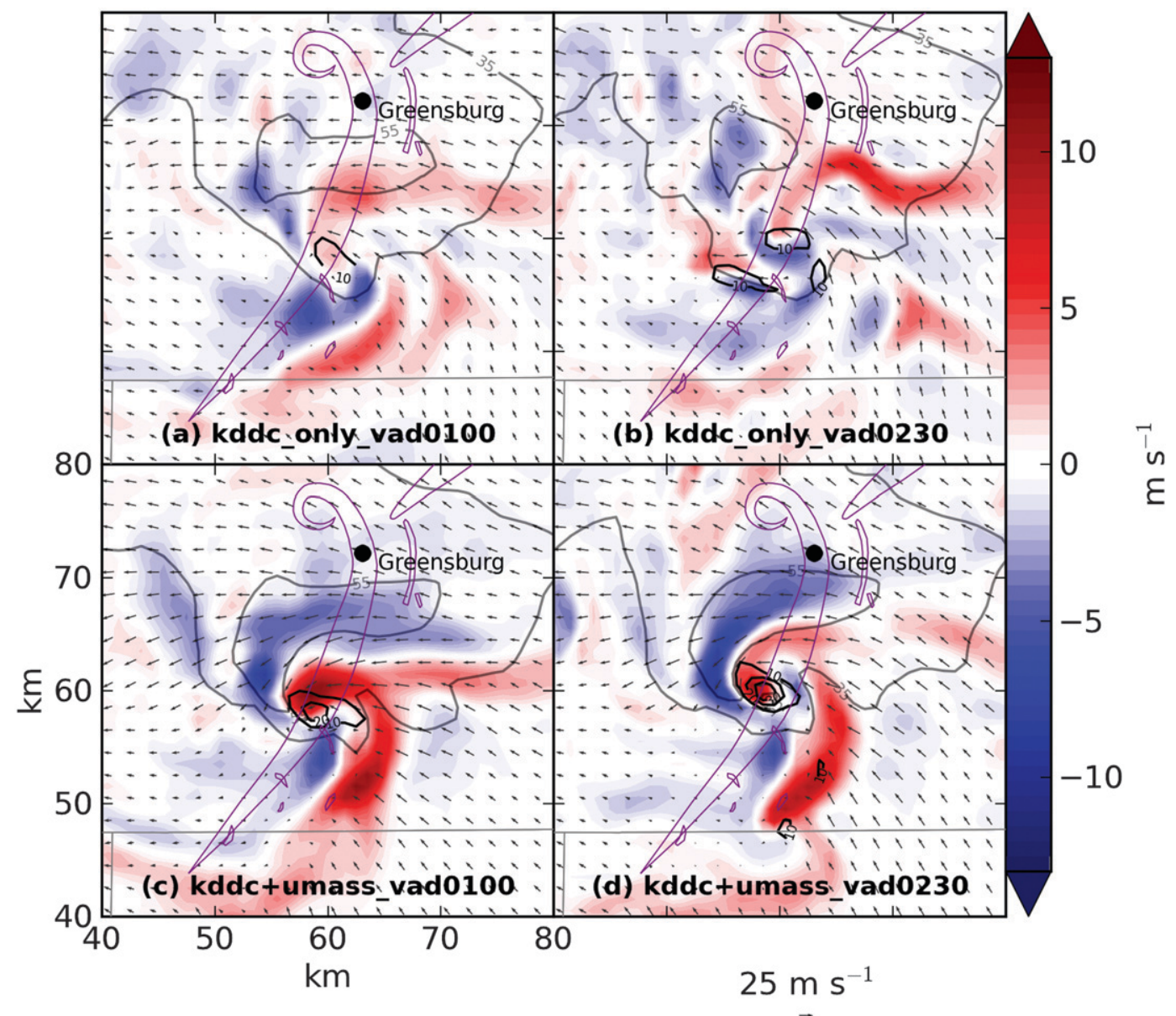

FIG. 10. As in Fig. 9, but slightly enlarged and showing prior ensemble mean vertical velocity (colored shading in $\mathrm{m} \mathrm{s}^{-1}$ ), vorticity (solid black contours in $10^{-3} \mathrm{~s}^{-1}$, starting from $10 \times 10^{-3} \mathrm{~s}^{-1}$ ), reflectivity (gray contours at 35 and $55 \mathrm{dBZ}$ ), and storm-relative velocity vectors (drawn every $2 \mathrm{~km}$ ).

where the difference between the observed reflectivity and the model reflectivity exceeded $30 \mathrm{~dB} Z$. Additive noise (described below) was also applied at this time.

After advancing the ensemble forward from 0100 to 0112 UTC, objectively analyzed radar observations were assimilated starting at 0112 UTC. A DA cycle period of 3 min was chosen as a compromise between the volume update time of KDDC (4.1 min) and that of UMass X-Pol ( $\sim 1 \mathrm{~min})$. During assimilation, $Z$ observations were used to update the $u, v, w$, and all hydrometeor mixing ratio fields (Dowell et al. 2011); $V_{r}$ observations were used to update the same fields along with the $\theta$ and water vapor mixing ratio $q_{v}$ fields.

An additive noise scheme (Caya et al. 2005; Dowell and Wicker 2009) was used to maintain ensemble spread throughout the assimilation period. Random noise was added to the model temperature $T$, dewpoint $T_{d}$, and horizontal velocity $(u, v)$ fields every 6 min in areas where KDDC $Z$ was greater than $20 \mathrm{~dB} Z$ during the preceding $6 \mathrm{~min}$. The standard deviations of the noise added to the $T, T_{d}, u$, and $v$ field (before smoothing) were prescribed as $1.0 \mathrm{~K}, 0.5 \mathrm{~K}, 2.0 \mathrm{~m} \mathrm{~s}^{-1}$, and $2.0 \mathrm{~m} \mathrm{~s}^{-1}$, respectively, and the horizontal (vertical) length scale for smoothing the perturbations was $4 \mathrm{~km}(2 \mathrm{~km})$.

The 3-min cycling of radar DA, 6-min cycling of additive noise, and 6-min cycling of small thermal bubblelike perturbations, as described above, were sufficient to establish the Greensburg storm in the model by 0130 UTC. After 0200 UTC, the thermal bubblelike perturbations were no longer used.

\section{f. Experiment nomenclature}

A total of four EnKF analysis experiments were performed (Table 3), combining two radar datasets and two 


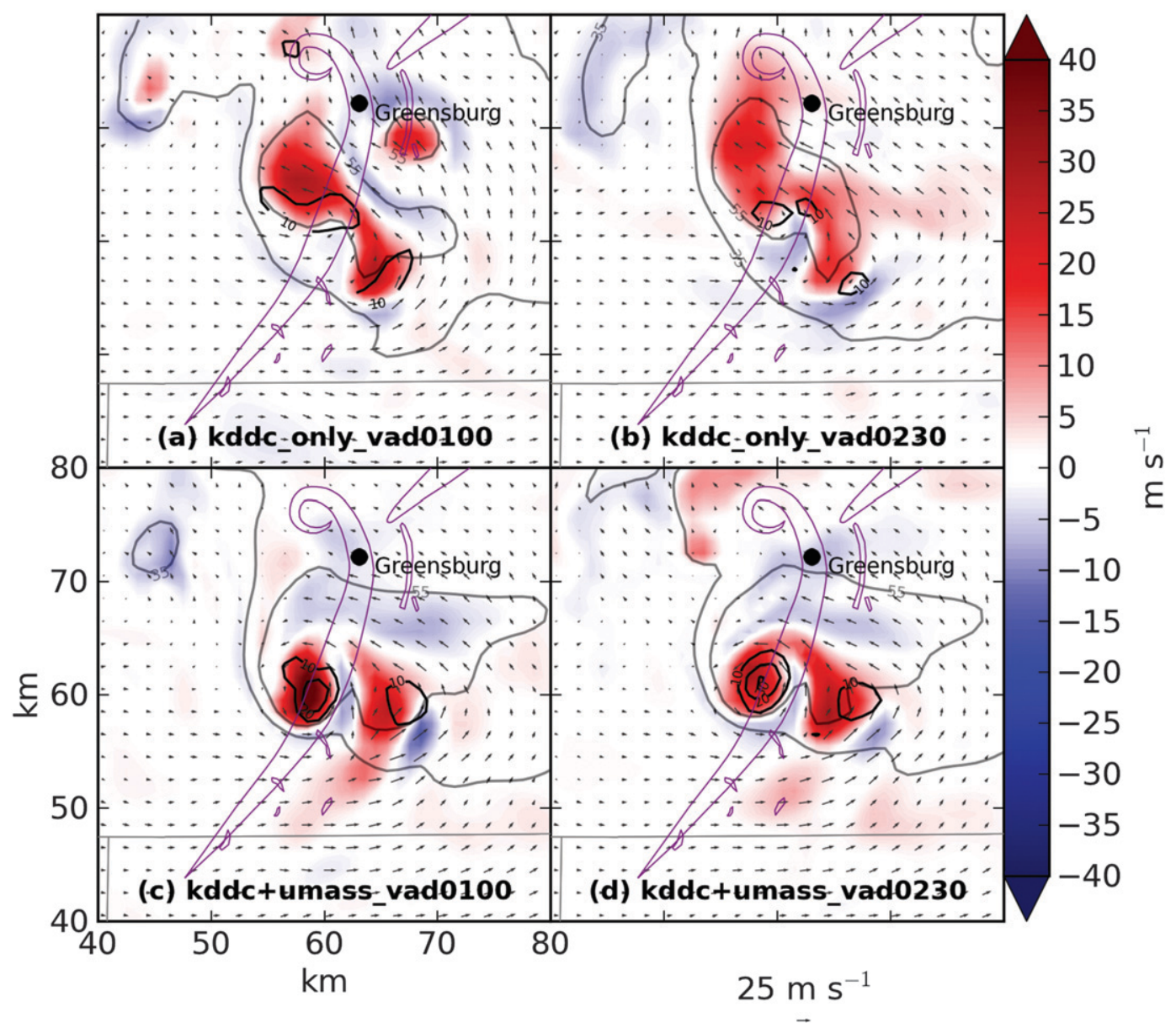

FIG. 11. As in Fig. 10, but at $5.3 \mathrm{~km}$ AGL.

wind profiles. In all four experiments, $\operatorname{KDDC} Z$ and $V_{r}$ data were assimilated (Fig. 7), and in two of the four experiments, supplemental UMass X-Pol $V_{r}$ data were also assimilated. These two sets of experiments are distinguished by the descriptive prefixes "kddc_only" and "kddc+umass." It can be seen in Figs. 7b and 7c that many more $V_{r}$ observations were assimilated in the kddc+umass experiments than in the kddc_only experiments, and that the number of $V_{r}$ observations varied with time, height, and depth of UMass X-Pol volumes (Fig. 2).

To represent the effect of the strengthening LLJ on the low-level wind profile, VAD wind profiles retrieved from KDDC $V_{r}$ data were incorporated into the initial velocity profile between the surface and $3 \mathrm{~km}$ AGL. In one set of experiments, the KDDC VAD at 0100 UTC (denoted by the suffix "vad0100"), prior to the onset of the LLJ, was used, while in the second, the KDDC VAD from 0230 UTC (suffix “vad0230”), containing a stronger LLJ, was used (Fig. 5).

\section{Results}

In all four experiments, a robust cyclonic supercell corresponding to the Greensburg storm was analyzed in the ensemble mean by 0130 UTC, indicating that the assimilation of KDDC data succeeded in establishing the rotating updraft and precipitation region of the

TABLE 3. Experiment naming convention.

\begin{tabular}{ccc}
\hline \hline Expt name & "Weaker LLJ" & "Stronger LLJ" \\
\hline KDDC $Z$ and $V_{r}$ & kddc_only_vad0100 & kddc_only_vad0230 \\
data assimilated & & \\
KDDC $Z$ and $V_{r}$ & kddc+umass_vad0100 & kddc+umass_vad0230 \\
and UMass $V_{r}$ & & \\
data assimilated & & \\
\hline
\end{tabular}


Greensburg storm (Figs. 8, 9, and 10). Assimilation of thinned, low $Z$ observations (Fig. 6a) suppressed spurious convection in the southern and eastern portions of the domain. An analyzed near-surface vorticity maximum closely followed the track of the Greensburg tornado in all ensemble members and can be seen in the ensemble mean (Fig. 10), while the midlevel (2-6 km AGL) rotating updraft was evident at $5.3 \mathrm{~km}$ AGL (Fig. 11). While many characteristics of the simulated storms were similar, the four experiments exhibited substantial differences, indicating that both the modification of the near-surface wind profile and the assimilation of the mobile Doppler radar data impacted the resulting analyses. These differences will be examined in more detail in sections $4 \mathrm{a}$ and $4 \mathrm{~b}$.

The reader is referred to Dowell and Wicker (2009) for detailed definitions of the observation-space diagnostic quantities innovation, root-mean-square of innovations (RMSI), spread, total spread, and consistency ratio $(\mathrm{CR})$, which we use to quantitatively evaluate the results. These quantities indicate the change in the model fields as a result of assimilation of observations and verify that sufficient spread is being maintained in the ensemble. In all four experiments, the CR of $V_{r}$ was less than 1 over most height layers (not shown) from 0112-0239 UTC (Fig. 12b), indicating too little model spread relative to the assumed observation errors. It appears that either our additive noise magnitudes (section 3e) may have been too small, that our assumed values for observation error variance (Table 2) were too small, or both. However, we believe that the individual ensemble members (not shown) exhibit sufficient variability for us to proceed with the analyses, and that the ensemble means are different enough from one another for meaningful information to be inferred from them.

\section{a. Assimilation of mobile Doppler radar data}

The assimilation of UMass X-Pol observations enabled detailed analysis of supercell features related to tornado production, including mesocyclones, updrafts, downdrafts, and surface boundaries such as the rearflank gust front. All of these features exhibited better definition in kddc+umass experiments than in kddc only experiments.

The kddc+umass experiments produced vortices (corresponding to tornadoes) that were stronger, deeper, and more persistent than those in corresponding kddc only experiments (in which UMass X-Pol data were withheld from assimilation; Figs. 10 and 11). These observations hold from the genesis through mature phases of the Greensburg tornado (i.e., 0200-0250 UTC), and can be seen in time-height plots of ensemble mean maximum vertical vorticity $(\zeta$, Fig. 13$)$ and vertical velocity $(w$, Fig. 14)
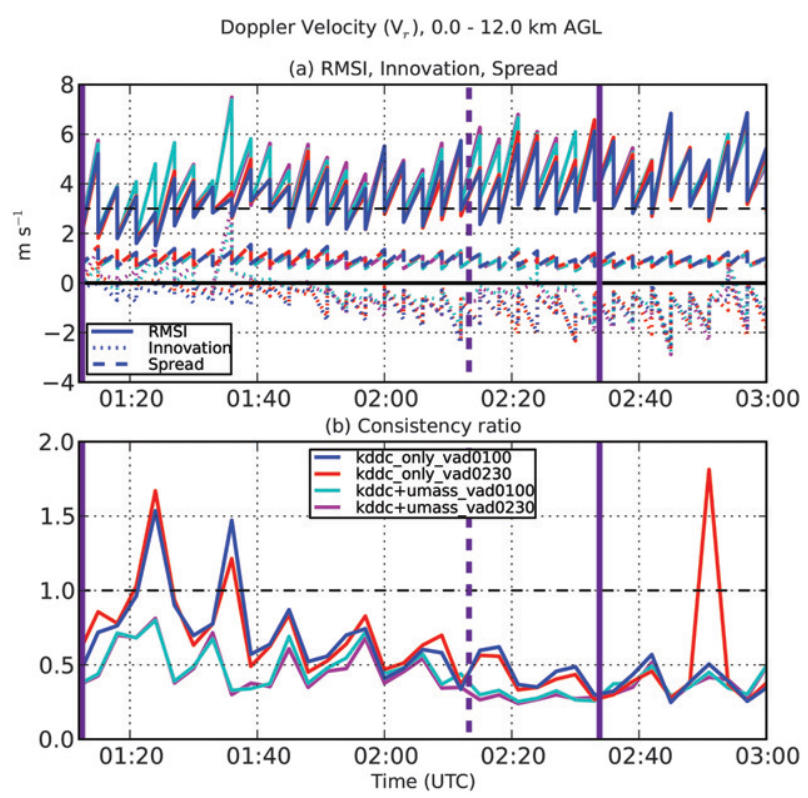

FIG. 12. The $V_{r}$ (a) RMSI (solid), mean innovation (dotted), and ensemble spread (dashed; $\mathrm{m} \mathrm{s}^{-1}$ ) and (b) consistency ratio (unitless). In (a), a horizontal dashed line marks $\sigma_{\mathrm{vr}}=3.0 \mathrm{~m} \mathrm{~s}^{-1}$. In (b), a horizontal dashed line marks unity. Vertical purple lines mark the start and end of UMass X-Pol volumetric data collection (Fig. 7); the dashed line marks when UMass X-Pol switched from collecting "shallow" to "deep" volumes.

in the area immediately around the Greensburg storm. Overall, maximum and average vertical vorticity, and average $w$, were larger in $\mathrm{kddc}+$ umass experiments than in corresponding kddc_only experiments (Figs. 13 and 14). Not only were more observations assimilated overall in the kddc+umass experiments than in the kddc_only experiments, but the assimilated UMass XPol $V_{r}$ observations were concentrated near the surface (Fig. 7), where the Greensburg tornado influenced the flow.

The most striking differences in the maximum vertical vorticity appear from 0213-0233 UTC, when "deep" UMass X-Pol volumes, which contained information throughout the depth of the mesocyclone(s), were assimilated in the kddc+umass experiments (Fig. 13). Evaluating the terms in the vertical vorticity tendency equation (e.g., Rotunno and Klemp 1985; Dowell and Bluestein 2002) near the surface, we found that the stretching term $[\zeta(\partial w / \partial z)]$ was negative near the Greensburg tornado vortex in kddc_only experiments, but positive in the kddc+umass experiments (Fig. 15). Positive near-surface horizontal convergence (and hence, positive vertical gradient of $w$, not shown) in the $\mathrm{kddc}+$ umass experiments was responsible for this difference; the sign of $\zeta$ in this area was positive in all four experiments. Areas of near-surface horizontal convergence 


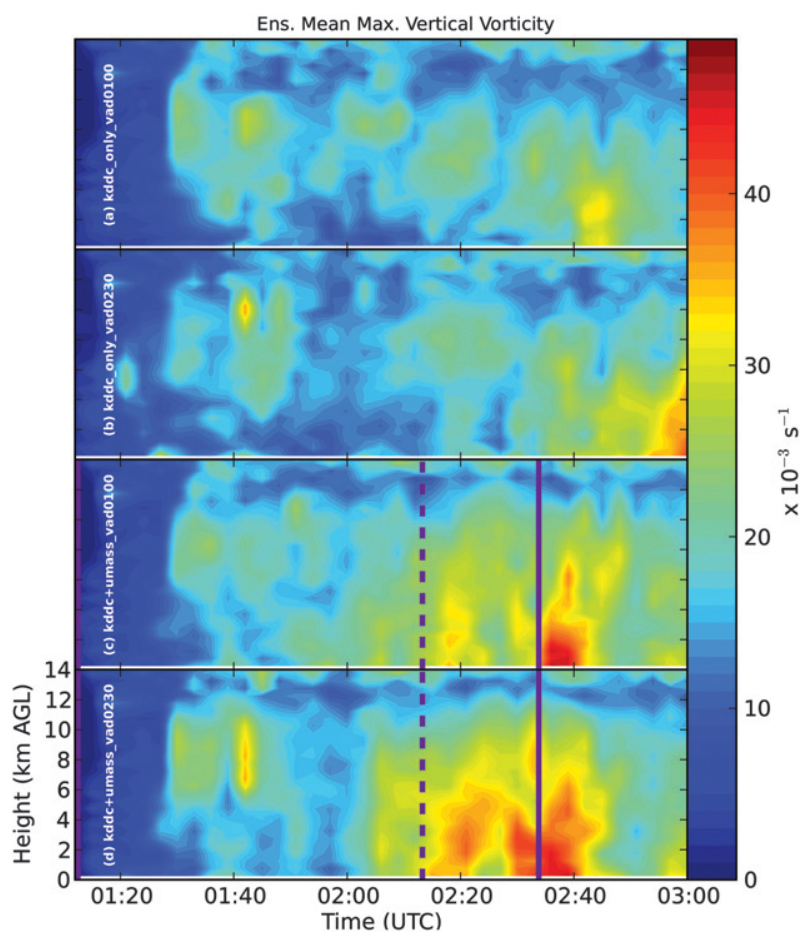

FIG. 13. Time-height plot of prior ensemble mean maximum vertical vorticity $\left(10^{-3} \mathrm{~s}^{-1}\right)$ for experiments (a) kddc_only vad0100, (b) kddc_only_vad0230, (c) kddc+umass_vad0100, and (d) kddc+umass_vad0230. Vertical purple lines mark changes in UMass X-Pol volumetric data collection as in Fig. 12.

(divergence) corresponded almost exactly with rising (sinking) motion (Fig. 10), so the horizontal convergence field is not shown. Some weaker vortices appearing between 0130 and 0145 UTC in the ensemble mean analyses of the kddc+umass_vad0230 experiment correspond to the incipient/remnant circulations of tornadoes 1-4 (Fig. 16). Tornadoes 1 through 4 were much smaller in scale $(<100$-m damage path width) and shorter-lived ( 4-13 min) than tornado 5 (Tanamachi et al. 2012). While the core flow in these earlier vortices would have been unresolvable at 1-km horizontal grid spacing, the vortices influenced the low-level flow fields on scales resolvable in the analyses. These vortices were less distinct in the kddc+umass_vad0100 experiment (not shown), and indistinguishable from noise in the kddc_only experiments (not shown). Similarly, vortices corresponding to tornadoes 9 and 10 were also analyzed in the ensemble mean (Fig. 17). However, vorticity maxima associated with tornadoes 6 through 8 (Fig. 2), all short lived ( $<2 \mathrm{~min}$ ), small ( $<100$-m damage path width) satellites of tornado 5 (Tanamachi et al. 2012), could not be detected in any of the ensemble mean analyses. We attribute the absence of analyzed vorticity maxima from tornadoes $6-8$ to their

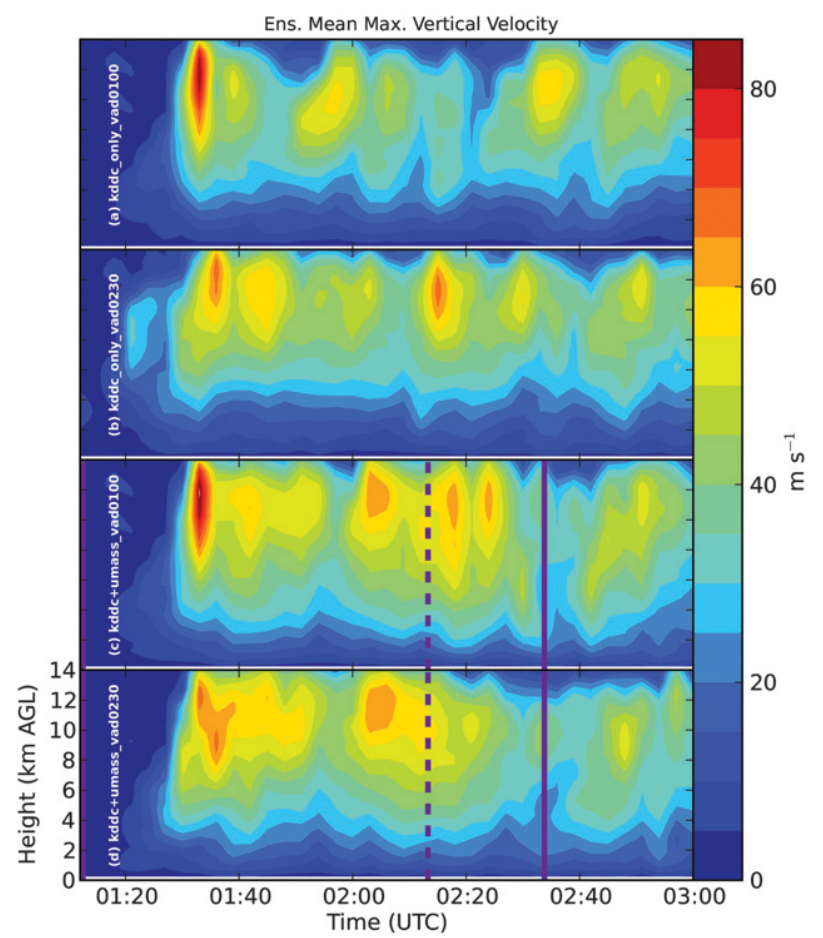

FIG. 14. As in Fig. 13, but for ensemble mean maximum vertical velocity $\left(\mathrm{m} \mathrm{s}^{-1}\right)$.

brevity; none lasted longer than a single UMass X-Pol volume scan ( $\sim 90 \mathrm{~s})$.

In both the kddc_only and kddc+umass experiments, the simulated Greensburg storms developed strong midlevel (2.0 to $6.0 \mathrm{~km} \mathrm{AGL)} \mathrm{updrafts} \mathrm{(Fig.} \mathrm{14).} \mathrm{Each}$ storm's midlevel updraft bifurcated into two updrafts between 0215 and 0221 UTC, with the Greensburg tornado's parent mesocyclone embedded in the western updraft (Fig. 11). The eastern updraft became stronger than the western updraft by 0239 UTC, causing the eastward propagation of the Greensburg storm and consequent rearward storm-relative motion of the Greensburg tornado (Dowell and Bluestein 2002). This change in the Greensburg tornado's storm-relative motion heralded the onset of its weakening phase, which continued until its demise at 0305 UTC northwest of Greensburg. The eastern updraft became the parent mesocyclone of a subsequent EF-3 tornado (T13 in Fig. 2) near Trousdale, Kansas (Lemon and Umscheid 2008). Although the location and timing of the updraft split are comparable between the experiments, stronger, more compact midlevel updrafts were analyzed in the kddc+umass experiments than in the kddc_only experiments (Fig. 11), corresponding with the assimilation of deep UMass X-Pol volumes (Fig. 7).

The analyzed low- and midlevel wind fields in the kddc+umass experiments continued to display major 
Ens. Mean Stretching, 05 May 2007 02:30:00, 750 m AGL

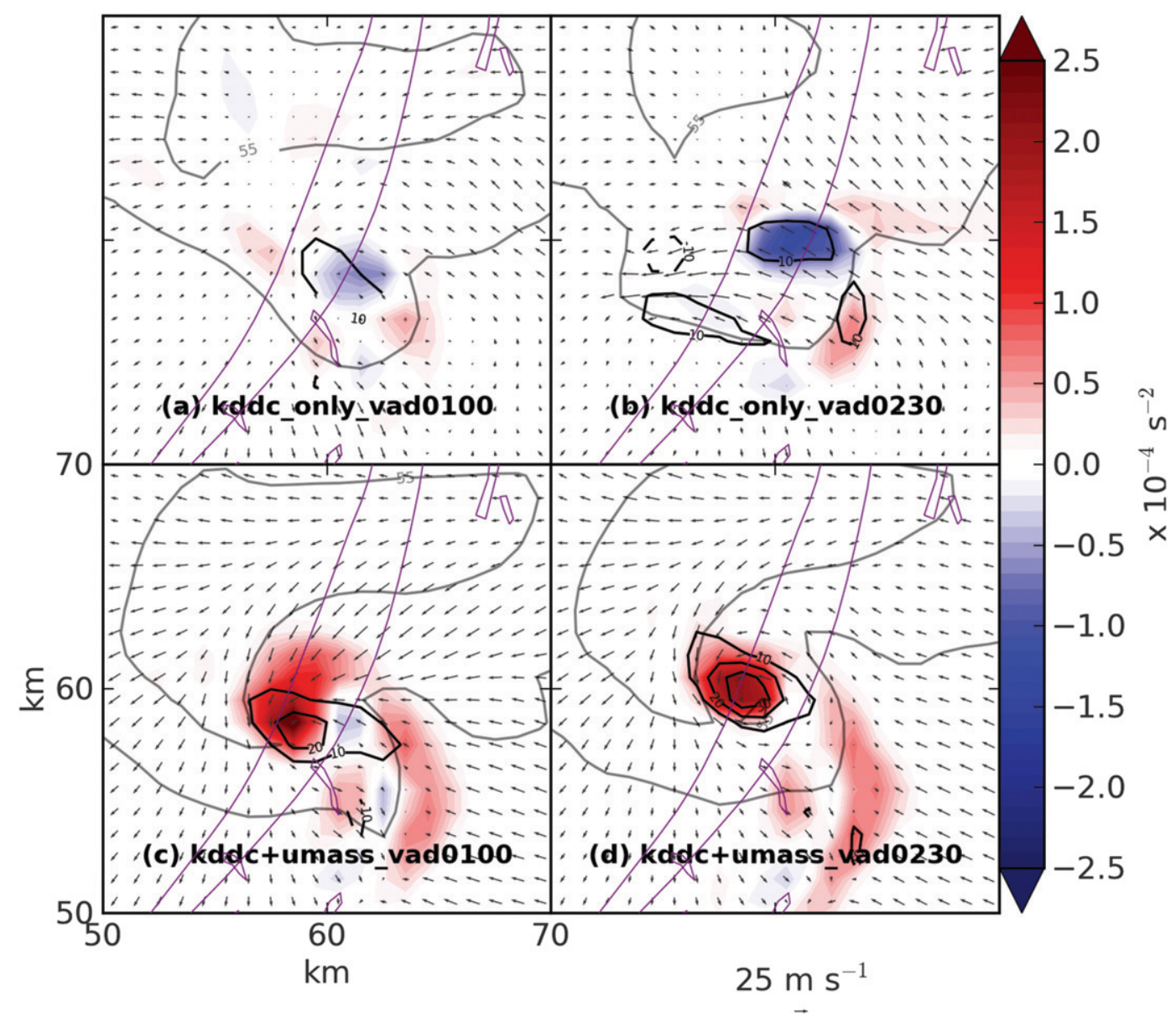

FIG. 15. As in Fig. 10, but showing the stretching term of the vertical vorticity tendency equation $\left(10^{-4} \mathrm{~s}^{-2}\right)$. Note that the panels are enlarged relative to those in Fig. 10, and that velocity vectors are drawn every $1 \mathrm{~km}$.

differences from those of the kddc_only experiments long after the UMass X-Pol data were no longer available for assimilation (i.e., 0234-0300 UTC). In particular, by 0245 UTC, the eastern updraft had developed a much stronger low-level mesocyclone in the kddc+ umass experiments than the kddc_only experiments (Fig. 18), signifying that the observations of low- and midlevel mesocyclone cycling contained in the UMass $\mathrm{X}$-Pol data continued to influence the model forecasts of the mesocyclones' evolution two or more cycles (i.e., 6 or more minutes) after their assimilation. This result concurs with those from Dawson et al. (2012), who found improvement in free forecasts of the Trousdale mesocyclone initialized at the start of or during the cycling process versus those initialized before the cycling began.

Expanding cold pools were produced in all four experiments, principally beneath the rear-flank downdraft of the Greensburg storm (Fig. 19). We caution against giving too much credence to the exact temperature values in the cold pools, as no suitable surface data were available for assimilation or verification. Cold pool strength is often strongly tied to the choice of microphysical parameterization scheme (e.g., single- vs multimoment, inclusion vs exclusion of ice species), and nonlinear feedback processes sometimes lead to vastly different cold pool structures, even when the same scheme is used with slightly different parameter values (Gilmore et al. 2004; Snook and Xue 2008; Dawson et al. 2010). We used a relatively simple, single-moment microphysical parameterization scheme (Table 2), but one that does include ice species. We focus on the structure of the cold pool, which we believe is informative.

At 0215 UTC, the cold pools (Fig. 19) exhibited a north-northwest-south-southeast-oriented boundary separating cooler air in the near-surface portion of the 

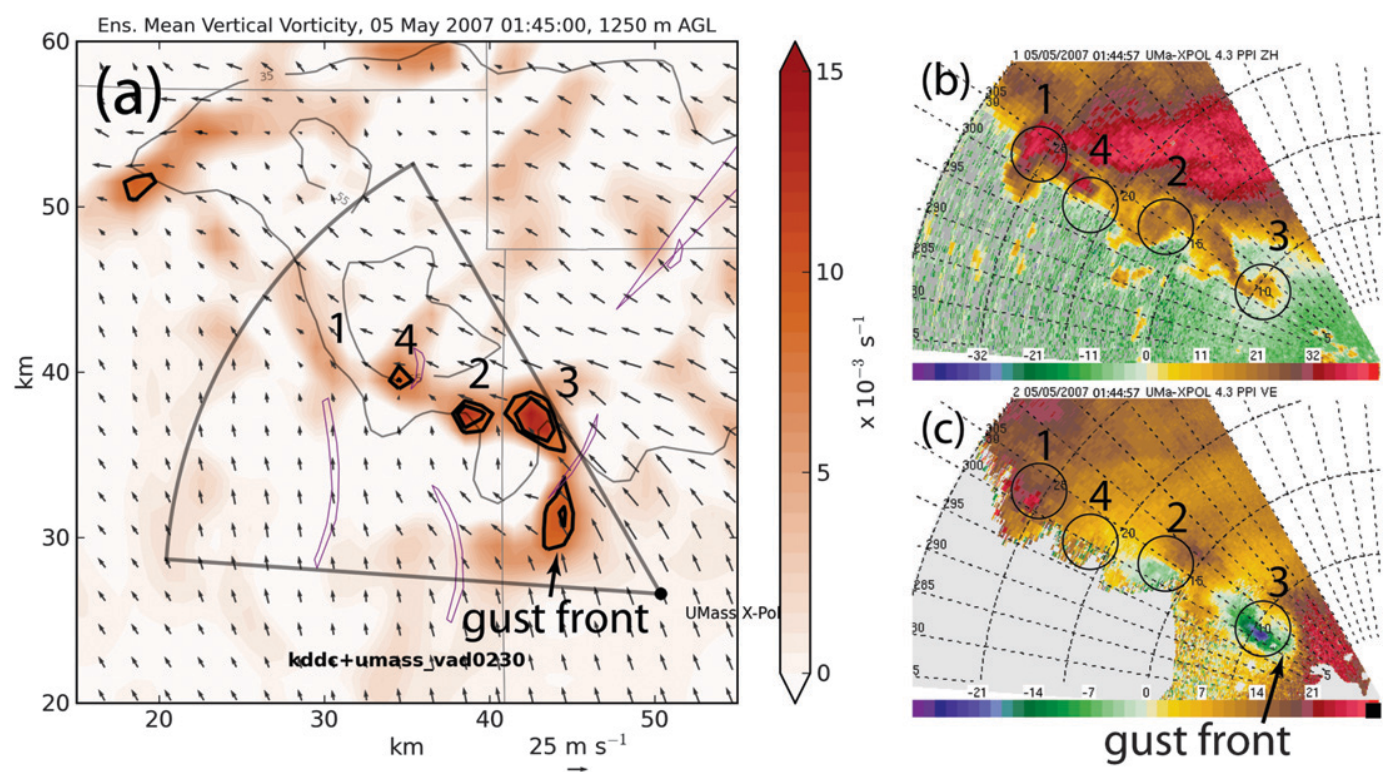

FIG. 16. (a) Ensemble mean analyzed vertical vorticity (colored shading), with solid black contours plotted at $8 \times$ $10^{-3} \mathrm{~s}^{-1}$ and $10 \times 10^{-3} \mathrm{~s}^{-1}$, reflectivity (gray contours at 35 and $55 \mathrm{dBZ}$ ), and storm-relative velocity vectors (plotted at 2-km intervals) at $1.3 \mathrm{~km}$ AGL for the kddc+umass_vad0230 experiment. Only positive values of vertical vorticity are plotted in (a), and the outline of the UMass X-Pol sector is overlaid. (b) UMass X-Pol uncalibrated reflectivity $(\mathrm{dBZ})$ and $(\mathrm{c})$ Doppler velocity $\left(\mathrm{m} \mathrm{s}^{-1}\right)$ at an elevation angle of $4.3^{\circ}$. Vertical vorticity maxima and circulations corresponding to tornadoes are circled and numbered as in Lemon and Umscheid (2008). At this time (0145 UTC), tornado 1 was dissipating, tornado 2 had already dissipated into a remnant vortex, tornado 3 was developing, and tornado 4 's vortex was forming above the $4.3^{\circ}$ elevation angle sweep surface.

rear-flank downdraft (RFD) of the Greensburg storm (similar to Fig. 10) from warmer air to its east. In the kddc+umass experiments, the magnitude of this temperature gradient is larger, and the accompanying nearsurface downdraft stronger, than in the kddc_only experiments. We conclude that the assimilation of the UMass X-Pol data makes both analyzed updrafts and downdrafts in the Greensburg storm stronger and more compact.

These changes to the updrafts and downdrafts are accompanied by changes to baroclinic vorticity generation in the Greensburg storm. Diagnosed baroclinic (solenoidal) generation of storm-relative streamwise horizontal vorticity (Adlerman et al. 1999) (Fig. 20) is particularly pronounced along the forward-flank gust front (where it is predominantly positive) and along the rear-flank gust front (where it is predominantly negative or antistreamwise). We can estimate the vorticity that might be acquired by a parcel traversing either of these areas. Consider the kddc+umass_0230vad experiment (Fig. 20d); a parcel moving westward at $20 \mathrm{~m} \mathrm{~s}^{-1}$ through the forward-flank baroclinic zone (which is about $15 \mathrm{~km}$ long and has a generation rate of $0.5 \times$ $10^{-4} \mathrm{~s}^{-2}$ ) could be expected to acquire about $4 \times$ $10^{-2} \mathrm{~s}^{-1}$ of streamwise horizontal vorticity, which could potentially be available for tilting into the vertical once the parcel reached the storm's updraft (Rotunno and Klemp 1985; Straka et al. 2007). A parcel moving northwestward at $10 \mathrm{~m} \mathrm{~s}^{-1}$ through the baroclinic zone along the along the rear-flank gust front (which is about $7 \mathrm{~km}$ long and has a generation rate of $1.0 \times 10^{-4} \mathrm{~s}^{-2}$ ) could be expected to acquire about of $7 \times 10^{-2} \mathrm{~s}^{-1}$ antistreamwise vorticity. We further speculate that if the parcel were subsequently drawn in toward the tornado, this antistreamwise vorticity could potentially be converted into positive vertical vorticity by downward tilting of the vortex lines in the RFD (Davies-Jones and Brooks 1993; Straka et al. 2007; Marquis et al. 2012). We emphasize that both of these scenarios are purely speculative; precise calculations of accumulated vorticity along trajectories that would be needed for confirmation are reserved for a future study incorporating a finer grid.

Assimilation of shallow UMass X-Pol volumes (01120213 UTC) was associated with increased RMSI (Fig. 12a) and corresponding decreases in the consistency ratio (Fig. 12b) total spread at and below $6 \mathrm{~km}$ relative to the kddc_only experiments (Fig. 21). When deep UMass X-Pol volumes were assimilated (0213-0233 UTC), these same effects extended throughout the model domain 

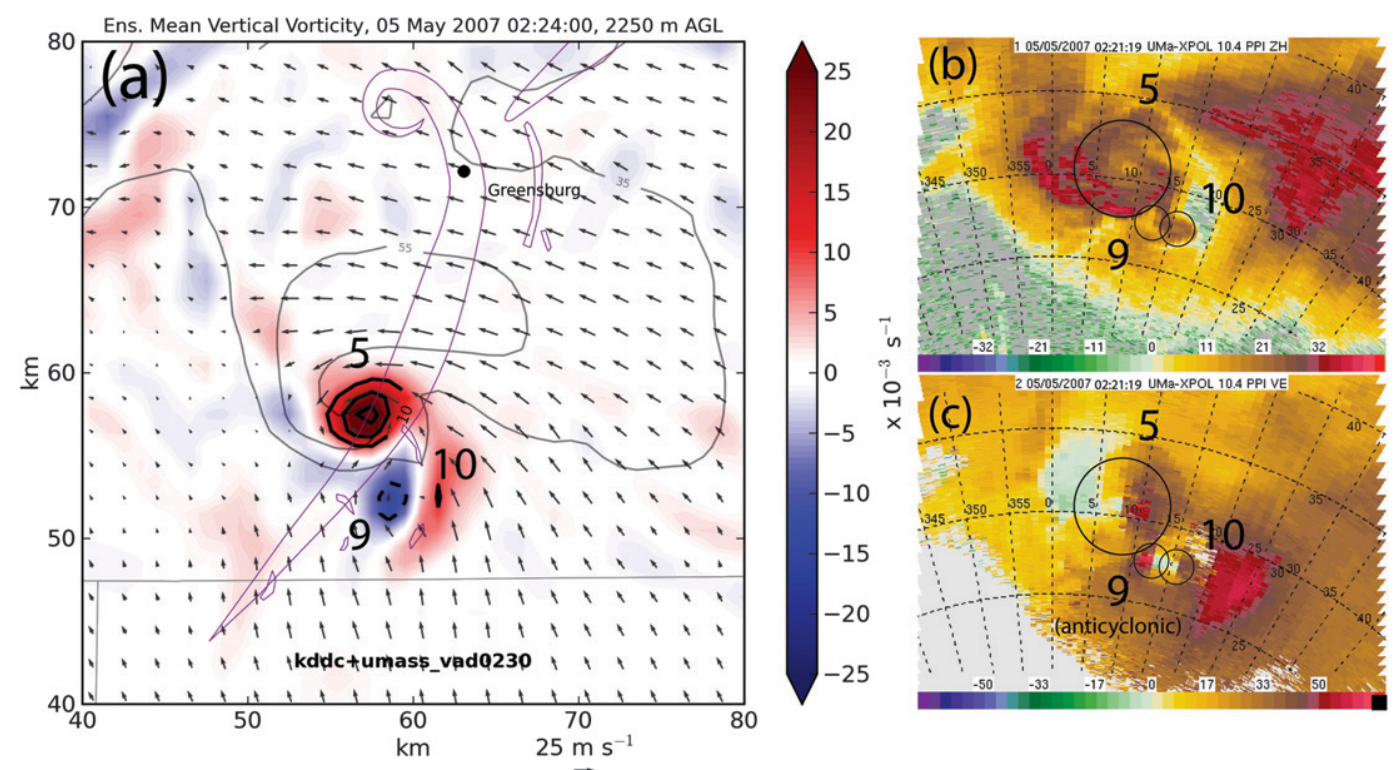

FIG. 17. (a) Ensemble mean analyzed vertical vorticity (colored shading) with solid black contours plotted at $-10 \times 10^{-3} \mathrm{~s}^{-1}$ (dashed) and at intervals of $10 \times 10^{-3} \mathrm{~s}^{-1}$ starting at $10 \times 10^{-3} \mathrm{~s}^{-1}$ (solid), reflectivity (gray contours at 35 and $55 \mathrm{dBZ}$ ), and storm-relative velocity vectors (plotted at 2-km intervals) at $2.3 \mathrm{~km}$ AGL at 0224 UTC for the kddc+ umass_vad0230 experiment. (b) UMass X-Pol uncalibrated reflectivity (dBZ) and (c) Doppler velocity $\left(\mathrm{m} \mathrm{s}^{-1}\right)$ at an elevation angle of $10.4^{\circ}$ at 0221 UTC. Vertical vorticity maxima and circulations corresponding to tornadoes are circled and numbered as in Lemon and Umscheid (2008). Note that tornado 9 was anticyclonic.

depth. We believe these differences in the observationspace diagnostics are directly attributable to the larger number of observations being assimilated in the $\mathrm{kddc}+$ umass experiments, which more strongly constrains the analyses.

Zhang et al. (2004), in perfect model experiments simulating a supercell, reported that assimilating synthetic low-altitude Doppler velocity observations, particularly those from and below $2 \mathrm{~km}$ AGL, improved the retrieved structures and strengths of updrafts, downdrafts, cold pools, and low-level vorticity maxima relative to experiments in which these data were withheld. Our results, using real observations, are largely consistent with theirs. Assimilation of more low-altitude Doppler velocity data (Fig. 7) resulted in more realistic retrievals of supercell features.

\section{b. Modification of initial 0-3-km wind profile}

The impacts of modifying of the initial $0-3-\mathrm{km}$ wind profile are less clear than those from assimilation of UMass X-Pol data. Differences between the vad0100 and vad0230 experiments include the reflectivity structures of the hook echoes (Fig. 8), the locations and intensities of vorticity maxima (Fig. 9) and $w$ maxima (Figs. 10 and 11), and cold pool structure (Fig. 19).

An initial updraft pulse (Fig. 14) at about 0135 UTC and $12 \mathrm{~km}$ AGL was stronger (about $85 \mathrm{~m} \mathrm{~s}^{-1}$ ) in the vad0100 experiments and weaker (about $68 \mathrm{~m} \mathrm{~s}^{-1}$ ) in the vad0230 experiments. This initial pulse occurred during the "spinup" period of the experiment (0100 0145 UTC), when the modeled storm (not shown) bore little resemblance to the real one. Because the real Greensburg storm (Fig. 1) developed its first intense ( $\geq 60 \mathrm{dBZ}$ ) reflectivity core in less than $4 \mathrm{~min}$ (between the 0107 and 0111 UTC KDDC volume scans), we consider the rapid development of the initial updraft pulse realistic.

To test the effects of the larger initial low-level shear on a single updraft, we conducted a simple, single warm bubble test in the vad0100 and vad0230 environments (Fig. 5). The resulting initial updraft pulse (not shown) was stronger in the vad0100 (weaker shear) environment than the vad0230 (stronger shear) environment. It appears that the weaker low-level shear in the vad0100 environment decreased entrainment into the updraft, allowing for a stronger initial updraft pulse (Weisman and Klemp 1982). Subsequent downdraft pulses (not shown) originating from the same altitude as the updraft pulse were also stronger in the vad0230 experiments than in the vad0100 experiments, possibly owing to increased entrainment and evaporation. Given the substantial differences in the simulated storms during the spinup period, it is not surprising that the ensemble mean fields are also different at all subsequent times. 
Ens. Mean Vertical Velocity, 05 May 2007 02:45:00, 2250 m AGL

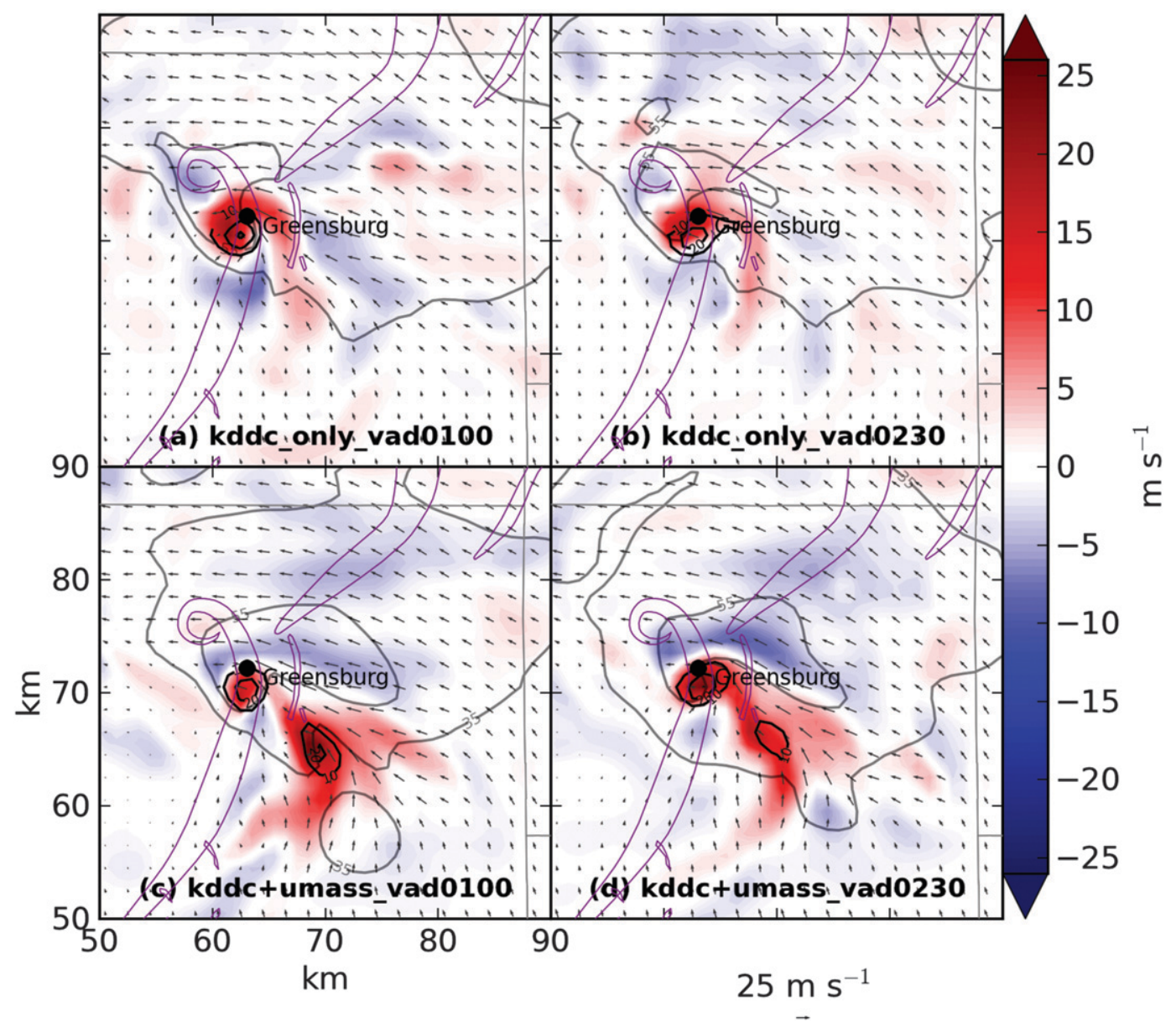

FIG. 18. As in Fig. 10, but at 0245 UTC, $2.3 \mathrm{~km} \mathrm{AGL,} \mathrm{and} \mathrm{shifted} \mathrm{slightly} \mathrm{northeast.}$

In the vad0230 experiments, the Greensburg storm produced more "warm" RFD pulses (in which $\theta$ was greater than or equal to the base-state temperature, 306.7 K; Fig. 19) than it did in the vad0100 experiments. Relatively warm RFDs have been associated with significant tornado production by supercells, possibly because they make more buoyant near-surface air available to be drawn into tornadoes (Markowski et al. 2002; Grzych et al. 2007). While more buoyant boundary layer air in the kddc+umass_vad0230 experiment could help to explain the stronger vorticity maximum when compared to the kddc+umass_vad0100 experiment, the vorticity maxima in the corresponding kddc_only experiments were comparable in strength with one another, and the impact of the low-level wind profile was less clear. The assimilation of UMass X-Pol data in combination with the larger low-level shear in the vad0230 environment appears to be the most conducive to the generation of strong vortices.
The vorticity maximum corresponding to the Greensburg tornado was stronger, on average, in the kddc+ umass_vad0230 experiment than in the kddc+umass vad0100 experiment (Figs. 10, 11, and 13c,d). One would expect the resulting enhancement of the downward-directed vertical perturbation pressure gradient force inside the stronger vortex to be associated with weaker updrafts in the kddc+umass vad0230 experiment (Fig. 14d) than in the kddc+ umass_vad0100 experiment (Fig. 14c), and it appears that this is indeed the case.

The vad0230 wind profile had greater low-level vertical wind shear (and hence, horizontal vorticity) than the vad0100 wind profile (Fig. 5). In the inflow sector, where the storm-relative flow was southeasterly, this horizontal vorticity was primarily streamwise at low levels (Fig. 22), and was continually replenished in the Greensburg storm's inflow sector from the relatively quiescent southeast quadrant of the model domain. This 
Ens. Mean Pert. Pot. Temp, 05 May 2007 02:15:00, 250 m AGL

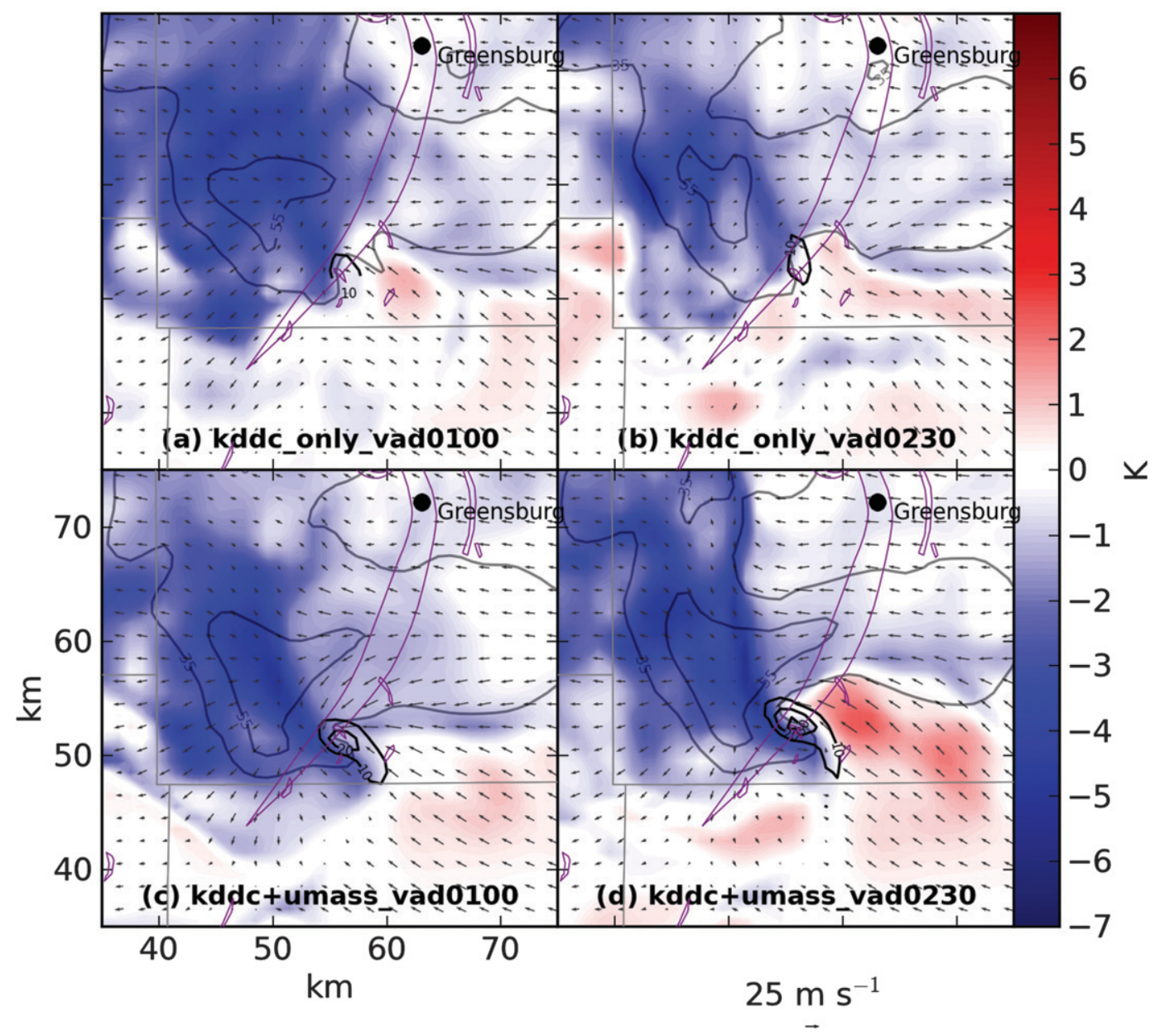

FIG. 19. Ensemble mean perturbation potential temperature (color shading, K) at $0.3 \mathrm{~km}$ AGL (the model level closest to the surface), vertical vorticity (solid black contours) at intervals of $10 \times 10^{-3} \mathrm{~s}^{-1}$ starting at $10 \times 10^{-3} \mathrm{~s}^{-1}$, reflectivity (gray contours at 35 and $55 \mathrm{dBZ}$ ), and storm-relative velocity vectors (plotted at $2 \mathrm{~km}$ intervals) at 0215 UTC. Potential temperatures are plotted relative to the initial model state $(\theta=306.7 \mathrm{~K})$.

streamwise vorticity would have been available for tilting into the vertical by rising motion in the updraft and/ or by uplift along the leading edge of the rear-flank gust front (Fig. 10). All other things being equal, this process could potentially lead to a stronger analyzed vortex, such as in the kddc+umass_vad0230 experiment (Wicker 1996; Shabbott and Markowski 2006). However, a recent study examining EnKF analyses of multiple tornadoes on finer grids $(500 \mathrm{~m})$ indicated that tilting of baroclinically generated horizontal vorticity may dominate tilting of environmental horizontal vorticity as a source of vertical vorticity in some tornadoes (Marquis et al. 2012). In our results, baroclinic generation of storm-relative streamwise horizontal vorticity is larger in the kddc+umass_vad0230 experiment than in the kddc+umass_vad0100 experiment (Fig. 20), lending some credibility to the above scenario. We await future studies in which forward-flank baroclinity in supercells is analyzed through the assimilation of thermodynamic measurements along with radar data, and more complex microphysical parameterization schemes are employed.

As noted in the previous subsection, vorticity maxima associated with some of the smaller, weaker tornadoes $(1-4,9$, and 10) were analyzed in the ensemble mean of the kddc+ umass_vad0230 experiment (Figs. 16 and 17), but were weaker or absent in the kddc+umass_vad0100 experiment (not shown). From these results, we infer that the inclusion of the LLJ in the initial vad0230 environment did positively impact the development and maintenance of vortices (relative to the vad0100 environment) throughout the experiment. 
Ens. Mean Streamwise Baroclinic Gen., 05 May 2007 02:15:00, 750 m AGL

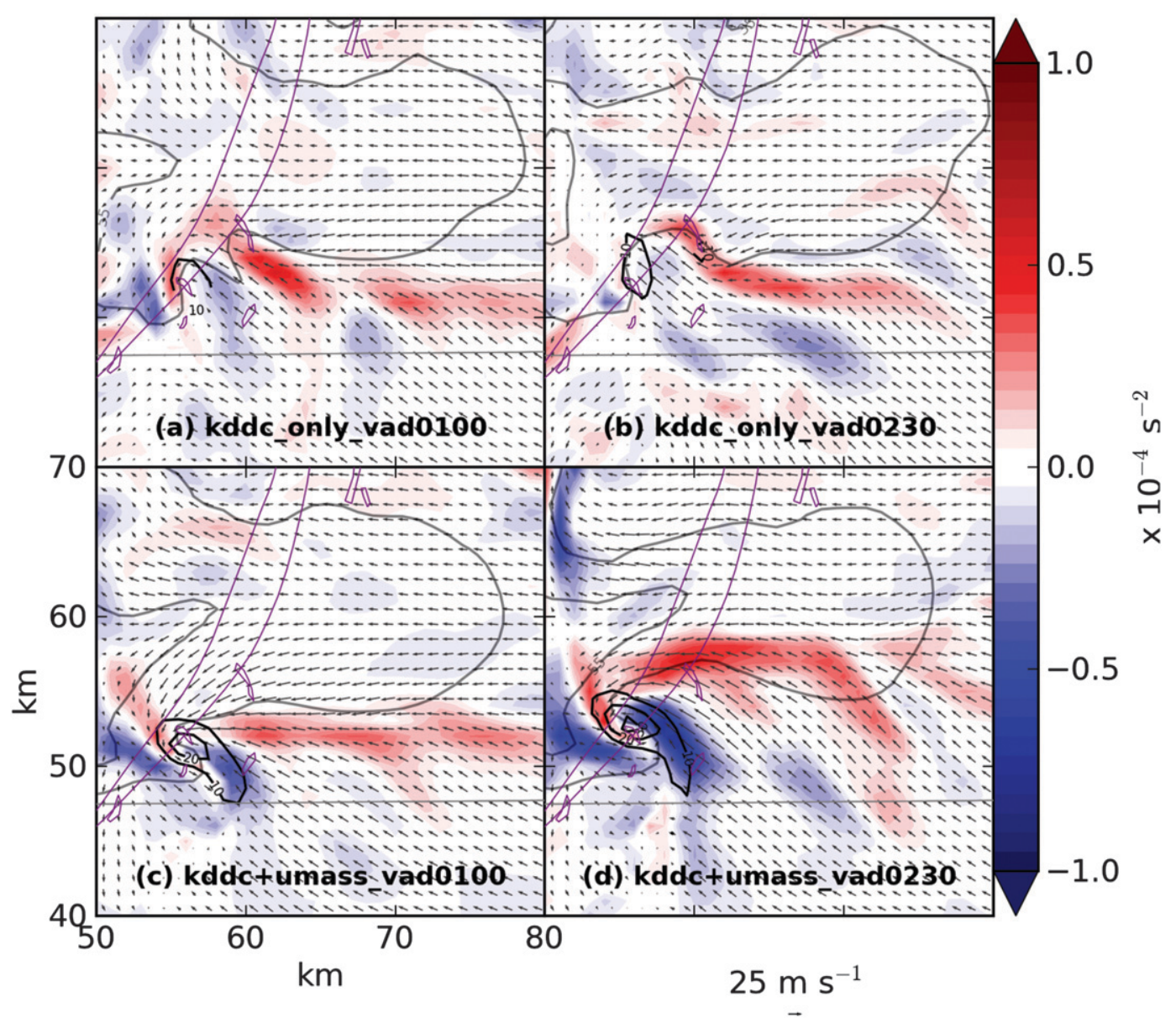

FIG. 20. As in Fig. 15, but showing the ensemble mean baroclinic generation of storm-relative streamwise horizontal vorticity $\left(10^{-4} \mathrm{~s}^{-2}\right)$ at 0215 UTC. Note that the panels are compressed and shifted relative to those in Fig. 15.

\section{Conclusions}

We evaluated the impacts on EnKF analyses of the Greensburg tornadic storm of assimilation of high-resolution, mobile radar data in an initially horizontally homogeneous environment. Overall, the additional assimilation of mobile Doppler radar data had a greater impact on the analyses than did modification of the low-level wind profile, even when these data were degraded to $1-\mathrm{km}$ horizontal grid spacing and assimilated in 3-min cycles. (For example, the kddc+ umass_vad0100 experiment was more similar to the kddc+umass_vad0230 experiment than the kddc only_vad0100 experiment.) However, both modifications made differences in the details of the analyzed storms, in particular the midlevel updraft strength and shape (Figs. 8, 9, and 11), cold pool structure (Fig. 19), gust front structure (Fig. 10), vortex strength (Figs. 10 and 13), the presence or absence of vorticity maxima associated with smaller vortices (Fig. 17), and baroclinic generation of horizontal vorticity (Fig. 20). All of these features were better defined in the analyses when UMass X-Pol data were assimilated rather than withheld.

Assimilation of UMass X-Pol data primarily impacted areas of the domain where wind fields had strong spatial gradients and were rapidly evolving (e.g., near gust fronts and intense vortices; see Fig. 10), resulting in substantial modifications to the inferred kinematics and dynamics of the Greensburg storm. While this finding is not entirely surprising, it underscores the relative importance of assimilating observations from multiple, independent platforms, particularly those collected at low altitudes (Zhang et al. 2004), when attempting to analyze a highly dynamic atmospheric system such as a tornadic supercell. These results thereby provide 


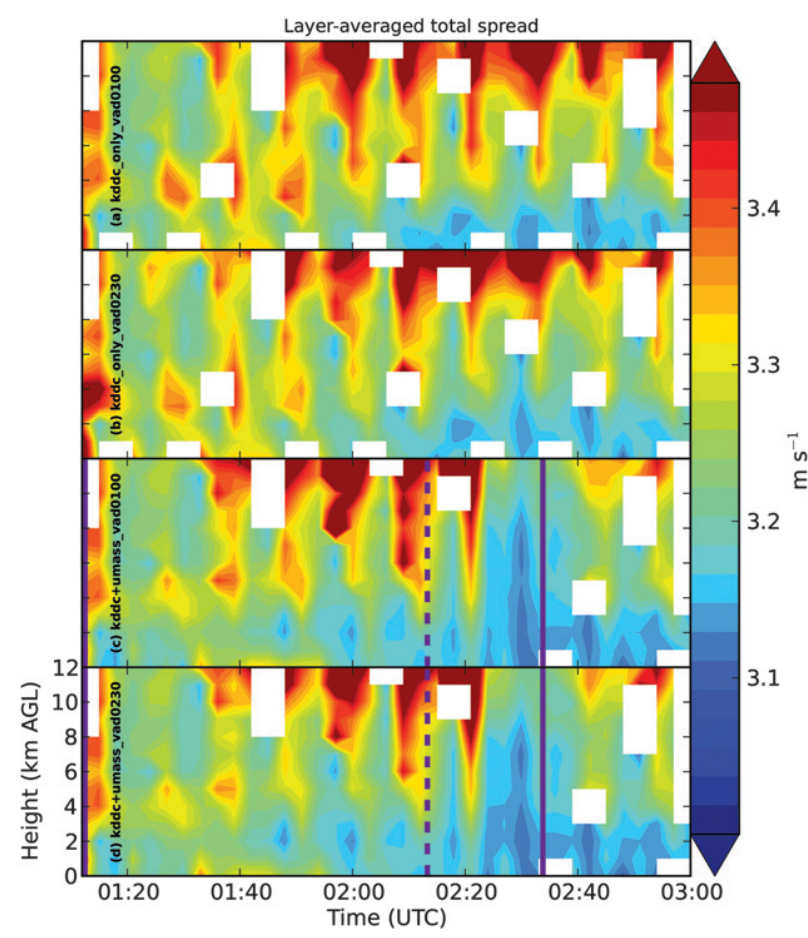

FIG. 21. Layer-averaged total spread $\left(\mathrm{m} \mathrm{s}^{-1}\right)$ over the entire domain for experiments (a) kddc_only_vad0100, (b) kddc_ only_vad0230, (c) kddc+umass_vad0100, and (d) kddc+umass_ vad0230. Vertical purple lines mark changes in UMass X-Pol volumetric data collection as in Fig. 12. White rectangles denote regions where a statistically insignificant number of observations were assimilated.

additional justification for efforts to collect radar observations in the $0-1-\mathrm{km}$ AGL layer, such as installing networks of closely spaced, short-range radars (Maki et al. 2008; McLaughlin et al. 2009), and field campaigns like the Second Verification of the Origins of Rotation in Tornadoes Experiment (VORTEX2; Wurman et al. 2012) that incorporate mobile Doppler radar deployment in severe storms.

Analyzed vortices and updrafts were stronger and deeper, especially when "deep" UMass X-Pol volumes were assimilated. In particular, the stronger horizontal convergence analyzed in the kddc+umass experiments resulted in a much stronger Greensburg tornado vortex. This outcome was linked to the sign of the stretching term in the vertical vorticity tendency equation, and, more tentatively, to enhanced baroclinic generation of horizontal vorticity along gust fronts. The effects of smaller, more transient vortices associated with tornadoes $1-4,9$, and 10 (which produced damage tracks $<100 \mathrm{~m}$ wide) appeared in the ensemble mean of the kddc+ umass_vad0230 experiment, but inconsistently in other experiments. These tornadoes and their parent

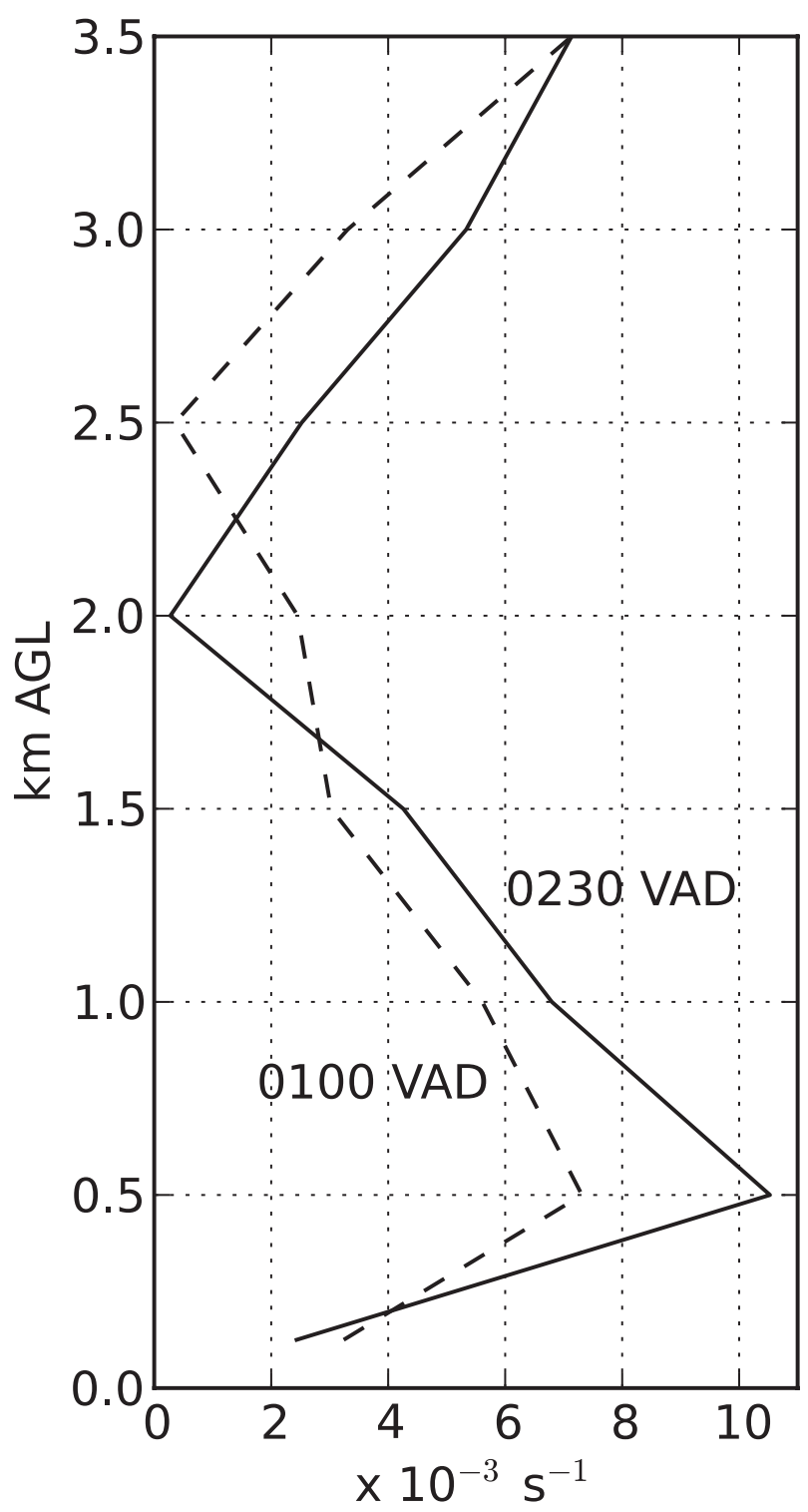

FIG. 22. Vertical profiles of storm-relative streamwise horizontal vorticity associated with the hodographs depicted in Fig. 5.

circulations clearly exert influence on scales resolvable in the analyses. The continued influence of the UMass X-Pol data assimilation was also evident in analyses after UMass X-Pol data were no longer available. In particular, a separate, eastern mesocyclone was stronger in the kddc+umass experiments than in the kddc_only experiments at 0245 UTC, more than $10 \mathrm{~min}$ after UMass X-Pol data collection ended.

There were also significant differences between the $\operatorname{vad} 0230$ and vad0100 analyses, particularly in the kddc+umass experiments. In particular, the analyzed Greensburg tornado vortex was stronger in the kddc+ umass_vad0230 analyses than in the kddc+ umass_vad0100 
analyses, indicating that the analyses were sensitive to the initial model environment for more than $90 \mathrm{~min}$ after assimilation of radar data began. We suggest that while the proper definition of the model initial environment and the assimilation of near-surface observations are both important, there may be an "optimal" combination of the two factors that is needed to achieve high-quality analysis of a supercell in an initially horizontally homogeneous framework. If the inflow sector is relatively pristine and free from convection (and therefore has lower scatter concentrations and fewer Doppler velocity observations available for assimilation), as it was in this case, the conditions there will remain relatively unmodified and continue to influence the development of the supercell throughout the assimilation period. These results are relevant since horizontally homogeneous initial environments are still used in many modeling studies of severe storms.

Our study focused primarily on the sensitivity of the analyses to different initial conditions and assimilated datasets. By repeating these experiments on a finer grid $(\sim 250 \mathrm{~m})$, saving model fields more frequently ( $\leq 1 \mathrm{~min}$ ), we hope to more fully exploit the high spatial and temporal resolution of the UMass X-Pol data to diagnose the circulation budget and vortex dynamics of the Greensburg storm's mesocyclones and tornadoes using trajectory analyses (e.g., Adlerman et al. 1999; Markowski et al. 2011a; Marquis et al. 2012). We anticipate that analysis and assimilation of data from novel radar systems, such as polarimetric, phased-array, and/or short-range, stationary, X-band radars (Jung et al. 2008b; Brewster et al. 2010; Yussouf and Stensrud 2010; Snook et al. 2011; Marquis et al. 2012) will permit fuller exploitation of a greatly expanded and diversified collection of radar systems in both research and forecasting.

Acknowledgments. This research was supported by National Science Foundation Grants ATM-0637148, ATM-0934307, and ATM-0802888, and by NOAA Grant NA08OAR4320904. Portions of this manuscript were completed while the first author was a graduate research assistant at the School of Meteorology and Cooperative Institute for Mesoscale Meteorological Studies, and subsequently, a postdoctoral research associate at the Center for Analysis and Prediction of Storms (CAPS) in Norman, Oklahoma. Sudie Kelly, Glen Romine, and Jeff Anderson at the National Center for Atmospheric Research (NCAR) in Boulder, Colorado, provided visiting scientist support and assistance with preliminary versions of these experiments.

The WSR-88D and NWS automated surface observing station data used in this study were obtained from the National Climatic Data Center (NCDC). Jeff Hutton of the NWS forecast office in Dodge City, Kansas, provided shape files for the damage tracks that appear in maps. Kery Hardwick, Vijay Venkatesh, and Dr. Stephen Frasier of MIRSL processed the UMass X-Pol data. Conversations with Drs. James Marquis, Chris Snyder, David Stensrud, and George Bryan were beneficial in the development of the experiments. Feedback from Dr. James Marquis and another anonymous reviewer strengthened and clarified the manuscript.

\section{REFERENCES}

Adlerman, E. J., K. K. Droegemeier, and R. Davies-Jones, 1999: A numerical simulation of cyclic mesocyclogenesis. J. Atmos. Sci., 56, 2045-2069.

Aksoy, A., D. C. Dowell, and C. Snyder, 2009: A multicase comparative assessment of the ensemble Kalman filter for assimilation of radar observations. Part I: Storm-scale analyses. Mon. Wea. Rev., 137, 1805-1824.

Bluestein, H. B., 2009: The formation and early evolution of the Greensburg, Kansas, tornadic supercell on 4 May 2007. Wea. Forecasting, 24, 899-920.

_ M. M. French, R. L. Tanamachi, S. Frasier, K. Hardwick, F. Junyent, and A. L. Pazmany, 2007: Close-range observations of tornadoes in supercells made with a dual-polarization, X-band, mobile Doppler radar. Mon. Wea. Rev., 135, 1522-1543.

Brewster, K., K. W. Thomas, J. Gao, J. Brotzge, M. Xue, and Y. Wang, 2010: A nowcasting system using full physics numerical weather prediction initialized with CASA and NEXRAD radar data. Preprints, 25th Conf. on Severe Local Storms, Denver, CO, Amer. Meteor. Soc., 9.4. [Available online at https:// ams.confex.com/ams/pdfpapers/176053.pdf.]

Brown, R. A., V. T. Wood, R. M. Steadham, R. R. Lee, B. A. Flickinger, and D. Sirmans, 2005: New WSR-88D Volume Coverage Pattern 12: Results of field tests. Wea. Forecasting, 20, 385-393.

Browning, K. A., and R. Wexler, 1968: The determination of kinematic properties of a wind field using Doppler radar. J. Appl. Meteor., 7, 105-113.

Caya, A., J. Sun, and C. Snyder, 2005: A comparison between the 4DVAR and the ensemble Kalman filter techniques for radar data assimilation. Mon. Wea. Rev., 133, 3081-3094.

Coniglio, M. C., D. J. Stensrud, and L. J. Wicker, 2006: Effects of upper-level shear on the structure and maintenance of strong quasi-linear mesoscale convective systems. J. Atmos. Sci., 63, 1231-1252.

Cressman, G. P., 1959: An operational objective analysis system. Mon. Wea. Rev., 87, 367-374.

Davies-Jones, R., and H. E. Brooks, 1993: Mesocyclogenesis from a theoretical perspective. The Tornado: Its Structure, Dynamics, Prediction, and Hazards, Geophys. Monogr., Vol. 79, Amer. Geophys. Union, 105-114.

Dawson, D. T., M. Xue, J. A. Milbrandt, and M. K. Yau, 2010: Comparison of evaporation and cold pool development between single-moment and multimoment bulk microphysics schemes in idealized simulations of tornadic thunderstorms Mon. Wea. Rev., 138, 1152-1171.

_ _ L. J. Wicker, E. R. Mansell, and R. L. Tanamachi, 2012: Impact of the environmental low-level wind profile on ensemble forecasts of the 4 May 2007 Greensburg, KS tornadic storm and associated mesocyclones. Mon. Wea. Rev., 140, 696-716. 
Doviak, R. J., and D. S. Zrnić, 1993: Doppler Weather Radar and Observations. 2nd ed. Academic Press, 562 pp.

Dowell, D. C., and H. B. Bluestein, 2002: The 8 June 1995 McLean, Texas, storm. Part II: Cyclic tornado formation, maintenance, and dissipation. Mon. Wea. Rev., 130, 2649-2670.

— semble data assimilation. J. Atmos. Oceanic Technol., 26, 911-927.

- — - and D. J. Stensrud, 2004a: High resolution analyses of the 8 May 2003 Oklahoma City storm. Part II: EnKF data assimilation and forecast experiments. Preprints, 22nd Conf. on Severe Local Storms, Hyannis, MA, Amer. Meteor. Soc., 12.5. [Available online at https://ams.confex.com/ams/pdfpapers/ 81393.pdf.]

_ , F. Zhang, L. J. Wicker, C. Snyder, and N. A. Crook, 2004b: Wind and temperature retrievals in the 17 May 1981 Arcadia, Oklahoma, supercell: Ensemble Kalman filter experiments. Mon. Wea. Rev., 132, 1982-2005.

— L L. J. Wicker, and C. Snyder, 2011: Ensemble Kalman filter assimilation of radar observations of the 8 May 2003 Oklahoma City supercell: Influences of reflectivity observations on storm-scale analyses. Mon. Wea. Rev., 139, 272-294.

Evensen, G., 1994: Sequential data assimilation with a nonlinear quasi-geostrophic model using Monte Carlo methods to forecast error statistics. J. Geophys. Res., 99, 10 143-10 162.

French, M. M., 2006: The 15 May 2003 Shamrock, Texas, supercell: A dual-Doppler analysis and EnKF data assimilation experiment. M.S. thesis, School of Meteorology, University of Oklahoma, $145 \mathrm{pp}$.

— , H. B. Bluestein, D. C. Dowell, L. J. Wicker, M. R. Kramar, and A. L. Pazmany, 2006: The 15 May 2003 Shamrock, Texas, supercell: A dual-Doppler analysis and EnKF dataassimilation experiment. Preprints, 23rd Conf. on Severe Local Storms, St. Louis, MO, Amer. Meteor. Soc., 14.16A. [Available online at https://ams.confex.com/ams/23SLS/webprogram/ Paper115156.html.]

Gaspari, G., and S. E. Cohn, 1999: Construction of correlation functions in two and three dimensions. Quart. J. Roy. Meteor. Soc., 125, 723-757.

Gilmore, M. S., J. M. Straka, and E. N. Rasmussen, 2004: Precipitation and evolution sensitivity in simulated deep convective storms: Comparisons between liquid-only and simple ice and liquid phase microphysics. Mon. Wea. Rev., 132,1897-1916.

Grzych, M. L., B. D. Lee, and C. A. Finley, 2007: Thermodynamic analysis of supercell rear-flank downdrafts from Project ANSWERS. Mon. Wea. Rev., 135, 240-246.

Houtekamer, P. L., and H. L. Mitchell, 1998: Data assimilation using an ensemble Kalman filter technique. Mon. Wea. Rev., 126, 796-811.

Jung, Y., G. Zhang, and M. Xue, 2008a: Assimilation of simulated polarimetric radar data for a convective storm using the ensemble Kalman filter. Part I: Observation operators for reflectivity and polarimetric variables. Mon. Wea. Rev., 136, 2228-2245.

__, M. Xue, G. Zhang, and J. M. Straka, 2008b: Assimilation of simulated polarimetric radar data for a convective storm using the ensemble Kalman filter. Part II: Impact of polarimetric data on storm analysis. Mon. Wea. Rev., 136, 22462260.

Kain, J. S., and Coauthors, 2010: Assessing advances in the assimilation of radar data and other mesoscale observations within a collaborative forecasting-research environment. Wea. Forecasting, 25, 1510-1521.
Lemon, L. R., and M. Umscheid, 2008: The Greensburg, KS tornadic storm: A storm of extremes. Preprints, 24th Conf. on Severe Local Storms, Savannah, GA, Amer. Meteor. Soc., 2.4. [Available online at https://ams.confex.com/ams/pdfpapers/ 141811.pdf.]

Lilly, D. K., 1990: Numerical prediction of thunderstorms-Has its time come? Quart. J. Roy. Meteor. Soc., 116, 779-798.

Lin, Y.-L., R. D. Farley, and H. D. Orville, 1983: Bulk parameterization of the snow field in a cloud model. J. Climate Appl. Meteor., 22, 1065-1092.

Maki, M., and Coauthors, 2008: X-band polarimetric radar network in the Tokyo metropolitan area-X-NET. Preprints, Fifth European Conf. on Radar Meteorology and Hydrology, Helsinki, Finland, Finnish Meteorological Institute, 5 pp.

Markowski, P. M., J. M. Straka, and E. N. Rasmussen, 2002: Direct surface thermodynamic observations within the rear-flank downdrafts of nontornadic and tornadic supercells. Mon. Wea. Rev., 130, 1692-1721.

_- Y. Richardson, J. Wurman, K. Kosiba, P. Robinson, and J. Marquis, 2011a: Observations from VORTEX2: The pretornadic phase of the Goshen County, Wyoming, supercell (5 June 2009). Preprints, 35th Conf. on Radar Meteorology, Pittsburgh, PA, Amer. Meteor. Soc., 8B.1. [Available online at https://ams.confex.com/ams/35Radar/webprogram/Manuscript/ Paper191881/radar35.pdf.]

-, M. Majcen, Y. Richardson, J. Marquis, and J. Wurman, 2011b: Characteristics of the wind field in a trio of nontornadic low-level mesocyclones observed by the Doppler On Wheels radars. Electron. J. Severe Storms Meteor., 6. [Available online at http://www.ejssm.org/ojs/index.php/ejssm/ article/view/75/63.]

Marquis, J., Y. Richardson, J. Wurman, and P. Markowski, 2008: Single- and dual-Doppler analysis of a tornadic vortex and surrounding storm-scale flow in the Crowell, Texas, supercell of 30 April 2000. Mon. Wea. Rev., 136, 5017-5043.

,-- , P. Markowski, D. C. Dowell, J. Wurman, K. Kosiba, and P. Robinson, 2010: Preliminary analysis of the Goshen County tornadic supercell on 5 June 2009 during VORTEX2 using EnKF assimilation of mobile radar and mesonet data. Preprints, 25th Conf. on Severe Local Storms, Denver, CO, Amer. Meteor. Soc., 6.7. [Available online at https://ams. confex.com/ams/pdfpapers/176182.pdf.]

,,--- D. Dowell, and J. Wurman, 2012: Tornado maintenance investigated with high-resolution dual-Doppler and EnKF analysis. Mon. Wea. Rev., 140, 3-27.

Marshall, J. S., and W. M. K. Palmer, 1948: The distribution of raindrops with size. J. Meteor., 5, 165-166.

McLaughlin, D., and Coauthors, 2009: Short-wavelength technology and the potential for distributed networks of small radar systems. Bull. Amer. Meteor. Soc., 90, 1797-1817.

Monfredo, W., 2008: Blown away in Greensburg, USA: Prediction and analysis of an EF-5 tornado. Weather, 63, 116-120.

National Climatic Data Center, cited 2009: Storm Events Database. [Available online at http://www.ncdc.noaa.gov/stormevents/.]

National Research Council, 1995: Assessment of Nexrad Coverage and Associated Weather Services. National Academies Press, 104 pp.

Oye, R., C. Mueller, and S. Smith, 1995: Software for radar translation, visualization, editing, and interpolation. Preprints, 27th Conf. on Radar Meteorology, Vail, CO, Amer. Meteor. Soc., 359-361.

Rotunno, R., and J. Klemp, 1985: On the rotation and propagation of simulated supercell thunderstorms. J. Atmos. Sci., 42, 271292. 
Shabbott, C. J., and P. M. Markowski, 2006: Surface in situ observations within the outflow of forward-flank downdrafts of supercell thunderstorms. Mon. Wea. Rev., 134, 1422-1441.

Snook, N. A., and M. Xue, 2008: Effects of microphysical drop size distribution on tornadogenesis in supercell thunderstorms. Geophys. Res. Lett., 35, L28403, doi:10.1029/2008GL035866.

- - - and Y. Jung, 2011: Analysis of a tornadic meoscale convective vortex assimilating CASA X-band and WSR-88D radar data using an ensemble Kalman filter. Mon. Wea. Rev., 139, 3446-3468.

Snyder, C., and F. Zhang, 2003: Assimilation of simulated Doppler radar observations with an ensemble Kalman filter. Mon. Wea. Rev., 131, 1663-1677.

Stensrud, D. J., and J. Gao, 2010: Importance of horizontally inhomogeneous environmental initial conditions to ensemble storm-scale radar data assimilation and very short-range forecasts. Mon. Wea. Rev., 138, 1250-1272.

— system. Bull. Amer. Meteor. Soc., 90, 1487-1499.

Straka, J. M., E. N. Rasmussen, R. P. Davies-Jones, and P. M. Markowski, 2007: An observational and idealized numerical examination of low-level counter-rotating vortices in the rear flank of supercells. Electron. J. Severe Storms Meteor., 2. [Available online at http://www.ejssm.org/ojs/index.php/ ejssm/article/view/32/33.]

Sun, J., 2005: Convective-scale assimilation of radar data: Progress and challenges. Quart. J. Roy. Meteor. Soc., 131, 3439-3463.

— perature analysis using single WSR-88D data. Wea. Forecasting, 16, 117-132.

Tanamachi, R. L., H. B. Bluestein, J. B. Houser, K. M. Hardwick, and S. J. Frasier, 2012: Mobile, X-band, polarimetric Doppler radar observations of the 4 May 2007 Greensburg, Kansas tornadic supercell. Mon. Wea. Rev., 140, 2103-2125.
Tong, M., and M. Xue, 2005: Ensemble Kalman filter assimilation of Doppler radar data with a compressible nonhydrostatic model: OSS experiments. Mon. Wea. Rev., 133, 1789-1807.

Weisman, M. L., and J. B. Klemp, 1982: The dependence of numerically simulated convective storms on vertical wind shear and buoyancy. Mon. Wea. Rev., 110, 504-520.

Whitaker, J. S., and T. M. Hamill, 2002: Ensemble data assimilation without perturbed observations. Mon. Wea. Rev., 130, 1913 1924.

Wicker, L. J., 1996: The role of near surface wind shear on the generation of low-level mesocyclones and tornadoes. Preprints, 18th Conf. on Severe Local Storms, San Francisco, CA, Amer. Meteor. Soc., 115-119.

_ elastic models using forward time schemes. Mon. Wea. Rev., 130, 2088-2097.

Wurman, J., K. Kosiba, P. Markowski, Y. Richardson, D. Dowell, and P. Robinson, 2010: Finescale single- and dual-Doppler analysis of tornado intensification, maintenance, and dissipation in the Orleans, Nebraska, supercell. Mon. Wea. Rev., 138, 4439-4455.

, D. Dowell, Y. Richardson, P. Markowski, E. Rasmussen, D. Burgess, L. Wicker, and H. B. Bluestein, 2012: The second Verification of the Origins of Rotation in Tornadoes Experiment: VORTEX2. Bull. Amer. Meteor. Soc., 93, 11471170.

Yussouf, N., and D. J. Stensrud, 2010: Impact of phased-array radar observations over a short assimilation period: Observing system simulation experiments using an ensemble Kalman filter. Mon. Wea. Rev., 138, 517-538.

Zhang, F., C. Snyder, and J. Sun, 2004: Impacts of initial estimate and observation availability on convective-scale data assimilation with an ensemble Kalman filter. Mon. Wea. Rev., 132, 1238-1253. 